\title{
ORB-SLAM: a Versatile and Accurate Monocular SLAM System
}

\author{
Raúl Mur-Artal*, J. M. M. Montiel, Member, IEEE, and Juan D. Tardós, Member, IEEE,
}

\begin{abstract}
This paper presents ORB-SLAM, a feature-based monocular SLAM system that operates in real time, in small and large, indoor and outdoor environments. The system is robust to severe motion clutter, allows wide baseline loop closing and relocalization, and includes full automatic initialization. Building on excellent algorithms of recent years, we designed from scratch a novel system that uses the same features for all SLAM tasks: tracking, mapping, relocalization, and loop closing. A survival of the fittest strategy that selects the points and keyframes of the reconstruction leads to excellent robustness and generates a compact and trackable map that only grows if the scene content changes, allowing lifelong operation. We present an exhaustive evaluation in 27 sequences from the most popular datasets. ORBSLAM achieves unprecedented performance with respect to other state-of-the-art monocular SLAM approaches. For the benefit of the community, we make the source code public.
\end{abstract}

Index Terms-Lifelong Mapping, Localization, Monocular Vision, Recognition, SLAM

\section{INTRODUCTION}

B UNDLE ADJUSTMENT (BA) is known to provide accurate estimates of camera localizations as well as a sparse geometrical reconstruction [1], [2], given that a strong network of matches and good initial guesses are provided. For long time this approach was considered unaffordable for real time applications such as Visual Simultaneous Localisation and Mapping (Visual SLAM). Visual SLAM has the goal of estimating the camera trajectory while reconstructing the environment. Nowadays we know that to achieve accurate results at non-prohibitive computational cost, a real time SLAM algorithm has to provide BA with:

- Corresponding observations of scene features (map points) among a subset of selected frames (keyframes).

- As complexity grows with the number of keyframes, their selection should avoid unnecessary redundancy.

- A strong network configuration of keyframes and points to produce accurate results, that is, a well spread set of keyframes observing points with significant parallax and with plenty of loop closure matches.

- An initial estimation of the keyframe poses and point locations for the non-linear optimization.

- A local map in exploration where optimization is focused to achieve scalability.

- The ability to perform fast global optimizations (e.g. pose graph) to close loops in real-time.

This work was supported by the Dirección General de Investigación of Spain under Project DPI2012-32168, the Ministerio de Educación Scholarship FPU13/04175 and Gobierno de Aragón Scholarship B121/13.

The authors are with the Instituto de Investigación en Ingeniería de Aragón (I3A), Universidad de Zaragoza, María de Luna 1, 50018 Zaragoza, Spain (e-mail: raulmur@unizar.es; josemari@unizar.es; tardos@unizar.es).

* Corresponding author.
The first real time application of BA was the visual odometry work of Mouragon et. al. [3], followed by the ground breaking SLAM work of Klein and Murray [4], known as Parallel Tracking and Mapping (PTAM). This algorithm, while limited to small scale operation, provides simple but effective methods for keyframe selection, feature matching, point triangulation, camera localization for every frame, and relocalization after tracking failure. Unfortunately several factors severely limit its application: lack of loop closing and adequate handling of occlusions, low invariance to viewpoint of the relocalization and the need of human intervention for map bootstrapping.

In this work we build on the main ideas of PTAM, the place recognition work of Gálvez-López and Tardós [5], the scale-aware loop closing of Strasdat et. al [6] and the use of covisibility information for large scale operation [7], [8], to design from scratch ORB-SLAM, a novel monocular SLAM system whose main contributions are:

- Use of the same features for all tasks: tracking, mapping, relocalization and loop closing. This makes our system more efficient, simple and reliable. We use ORB features [9] which allow real-time performance without GPUs, providing good invariance to changes in viewpoint and illumination.

- Real time operation in large environments. Thanks to the use of a covisibility graph, tracking and mapping is focused in a local covisible area, independent of global map size.

- Real time loop closing based on the optimization of a pose graph that we call the Essential Graph. It is built from a spanning tree maintained by the system, loop closure links and strong edges from the covisibility graph.

- Real time camera relocalization with significant invariance to viewpoint and illumination. This allows recovery from tracking failure and also enhances map reuse.

- A new automatic and robust initialization procedure based on model selection that permits to create an initial map of planar and non-planar scenes.

- A survival of the fittest approach to map point and keyframe selection that is generous in the spawning but very restrictive in the culling. This policy improves tracking robustness, and enhances lifelong operation because redundant keyframes are discarded.

We present an extensive evaluation in popular public datasets from indoor and outdoor environments, including hand-held, car and robot sequences. Notably, we achieve better camera localization accuracy than the state of the art in direct methods [10], which optimize directly over pixel intensities instead of feature reprojection errors. We include a discussion 
in Section IX-B on the possible causes that can make featurebased methods more accurate than direct methods.

The loop closing and relocalization methods here presented are based on our previous work [11]. A preliminary version of the system was presented in [12]. In the current paper we add the initialization method, the Essential Graph, and perfect all methods involved. We also describe in detail all building blocks and perform an exhaustive experimental validation.

To the best of our knowledge, this is the most complete and reliable solution to monocular SLAM, and for the benefit of the community we make the source code public. Demonstration videos and the code can be found in our project webpage ${ }^{1}$.

\section{RELATED WORK}

\section{A. Place Recognition}

The survey by Williams et al. [13] compared several approaches for place recognition and concluded that techniques based on appearance, that is image to image matching, scale better in large environments than map to map or image to map methods. Within appearance based methods, bags of words techniques [14], such as the probabilistic approach FAB-MAP [15], are to the fore because of their high efficiency. DBoW2 [5] used for the first time bags of binary words obtained from BRIEF descriptors [16] along with the very efficient FAST feature detector [17]. This reduced in more than one order of magnitude the time needed for feature extraction, compared to SURF [18] and SIFT [19] features that were used in bags of words approaches so far. Although the system demonstrated to be very efficient and robust, the use of BRIEF, neither rotation nor scale invariant, limited the system to in-plane trajectories and loop detection from similar viewpoints. In our previous work [11], we proposed a bag of words place recognizer built on DBoW2 with ORB [9]. ORB are binary features invariant to rotation and scale (in a certain range), resulting in a very fast recognizer with good invariance to viewpoint. We demonstrated the high recall and robustness of the recognizer in four different datasets, requiring less than $39 \mathrm{~ms}$ (including feature extraction) to retrieve a loop candidate from a $10 \mathrm{~K}$ image database. In this work we use an improved version of that place recognizer, using covisibility information and returning several hypotheses when querying the database instead of just the best match.

\section{B. Map Initialization}

Monocular SLAM requires a procedure to create an initial map because depth cannot be recovered from a single image. One way to solve the problem is to initially track a known structure [20]. In the context of filtering approaches, points can be initialized with high uncertainty in depth using an inverse depth parametrization [21], which hopefully will later converge to their real positions. The recent semi-dense work of Engel et al. [10], follows a similar approach initializing the depth of the pixels to a random value with high variance.

Initialization methods from two views either assumes locally scene planarity [4], [22] and recover the relative camera pose

\footnotetext{
${ }^{1}$ http://webdiis.unizar.es/ raulmur/orbslam
}

from a homography using the method of Faugeras et. al [23], or compute an essential matrix [24], [25] that models planar and general scenes, using the five-point algorithm of Nister [26], which requires to deal with multiple solutions. Both reconstruction methods are not well constrained under low parallax and suffer from a twofold ambiguity solution if all points of a planar scene are closer to one of the camera centers [27]. On the other hand if a non-planar scene is seen with parallax a unique fundamental matrix can be computed with the eight-point algorithm [2] and the relative camera pose can be recovered without ambiguity.

We present in Section IV a new automatic approach based on model selection between a homography for planar scenes and a fundamental matrix for non-planar scenes. A statistical approach to model selection was proposed by Torr et al. [28]. Under a similar rationale we have developed a heuristic initialization algorithm that takes into account the risk of selecting a fundamental matrix in close to degenerate cases (i.e. planar, nearly planar, and low parallax), favoring the selection of the homography. In the planar case, for the sake of safe operation, we refrain from initializing if the solution has a twofold ambiguity, as a corrupted solution could be selected. We delay the initialization until the method produces a unique solution with significant parallax.

\section{Monocular SLAM}

Monocular SLAM was initially solved by filtering [20], [21], [29], [30]. In that approach every frame is processed by the filter to jointly estimate the map feature locations and the camera pose. It has the drawbacks of wasting computation in processing consecutive frames with little new information and the accumulation of linearization errors. On the other hand keyframe-based approaches [3], [4] estimate the map using only selected frames (keyframes) allowing to perform more costly but accurate bundle adjustment optimizations, as mapping is not tied to frame-rate. Strasdat et. al [31] demonstrated that keyframe-based techniques are more accurate than filtering for the same computational cost.

The most representative keyframe-based SLAM system is probably PTAM by Klein and Murray [4]. It was the first work to introduce the idea of splitting camera tracking and mapping in parallel threads, and demonstrated to be successful for real time augmented reality applications in small environments. The original version was later improved with edge features, a rotation estimation step during tracking and a better relocalization method [32]. The map points of PTAM correspond to FAST corners matched by patch correlation. This makes the points only useful for tracking but not for place recognition. In fact PTAM does not detect large loops, and the relocalization is based on the correlation of low resolution thumbnails of the keyframes, yielding a low invariance to viewpoint.

Strasdat et. al [6] presented a large scale monocular SLAM system with a front-end based on optical flow implemented on a GPU, followed by FAST feature matching and motiononly $B A$, and a back-end based on sliding-window BA. Loop closures were solved with a pose graph optimization with similarity constraints (7DoF), that was able to correct the scale 
drift appearing in monocular SLAM. From this work we take the idea of loop closing with 7DoF pose graph optimization and apply it to the Essential Graph defined in Section III-D

Strasdat et. al [7] used the front-end of PTAM, but performed the tracking only in a local map retrieved from a covisibility graph. They proposed a double window optimization back-end that continuously performs BA in the inner window, and pose graph in a limited-size outer window. However, loop closing is only effective if the size of the outer window is large enough to include the whole loop. In our system we take advantage of the excellent ideas of using a local map based on covisibility, and building the pose graph from the covisibility graph, but apply them in a totally redesigned frontend and back-end. Another difference is that, instead of using specific features for loop detection (SURF), we perform the place recognition on the same tracked and mapped features, obtaining robust frame-rate relocalization and loop detection.

Pirker et. al [33] proposed CD-SLAM, a very complete system including loop closing, relocalization, large scale operation and efforts to work on dynamic environments. However map initialization is not mentioned. The lack of a public implementation does not allow us to perform a comparison of accuracy, robustness or large-scale capabilities.

The visual odometry of Song et al. [34] uses ORB features for tracking and a temporal sliding window BA back-end. In comparison our system is more general as they do not have global relocalization, loop closing and do not reuse the map. They are also using the known distance from the camera to the ground to limit monocular scale drift.

Lim et. al [25], work published after we submitted our preliminary version of this work [12], use also the same features for tracking, mapping and loop detection. However the choice of BRIEF limits the system to in-plane trajectories. Their system only tracks points from the last keyframe so the map is not reused if revisited (similar to visual odometry) and has the problem of growing unbounded. We compare qualitatively our results with this approach in section VIII-E.

The recent work of Engel et. al [10], known as LSDSLAM, is able to build large scale semi-dense maps, using direct methods (i.e. optimization directly over image pixel intensities) instead of bundle adjustment over features. Their results are very impressive as the system is able to operate in real time, without GPU acceleration, building a semi-dense map, with more potential applications for robotics than the sparse output generated by feature-based SLAM. Nevertheless they still need features for loop detection and their camera localization accuracy is significantly lower than in our system and PTAM, as we show experimentally in Section VIII-B. This surprising result is discussed in Section IX-B.

In a halfway between direct and feature-based methods is the semi-direct visual odometry SVO of Forster et al. [22]. Without requiring to extract features in every frame they are able to operate at high frame-rates obtaining impressive results in quadracopters. However no loop detection is performed and the current implementation is mainly thought for downward looking cameras.

Finally we want to discuss about keyframe selection. All visual SLAM works in the literature agree that running BA

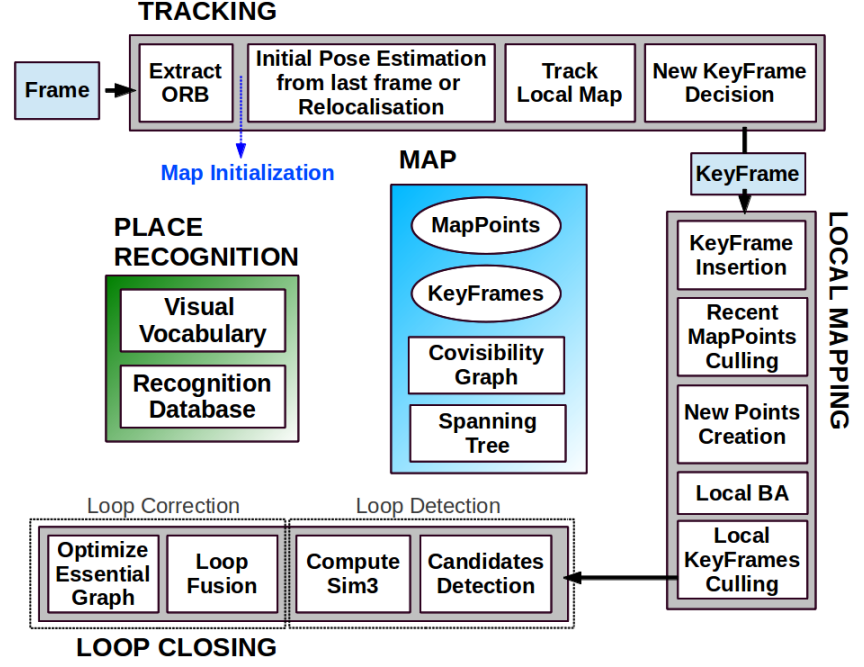

Fig. 1. ORB-SLAM system overview, showing all the steps performed by the tracking, local mapping and loop closing threads. The main components of the place recognition module and the map are also shown.

with all the points and all the frames is not feasible. The work of Strasdat et al. [31] showed that the most costeffective approach is to keep as much points as possible, while keeping only non-redundant keyframes. The PTAM approach was to insert keyframes very cautiously to avoid an excessive growth of the computational complexity. This restrictive keyframe insertion policy makes the tracking fail in hard exploration conditions. Our survival of the fittest strategy achieves unprecedented robustness in difficult scenarios by inserting keyframes as quickly as possible, and removing later the redundant ones, to avoid the extra cost.

\section{SYSTEM OVERVIEW}

\section{A. Feature Choice}

One of the main design ideas in our system is that the same features used by the mapping and tracking are used for place recognition to perform frame-rate relocalization and loop detection. This makes our system efficient and avoids the need to interpolate the depth of the recognition features from near SLAM features as in previous works [6], [7]. We requiere features that need for extraction much less than $33 \mathrm{~ms}$ per image, which excludes the popular SIFT $(\sim 300 \mathrm{~ms})$ [19], SURF $(\sim 300 \mathrm{~ms})$ [18] or the recent A-KAZE $(\sim 100 \mathrm{~ms})$ [35]. To obtain general place recognition capabilities, we require rotation invariance, which excludes BRIEF [16] and LDB [36].

We chose ORB [9], which are oriented multi-scale FAST corners with a 256 bits descriptor associated. They are extremely fast to compute and match, while they have good invariance to viewpoint. This allows to match them from wide baselines, boosting the accuracy of BA. We already shown the good performance of ORB for place recognition in [11]. While our current implementation make use of ORB, the techniques proposed are not restricted to these features.

\section{B. Three Threads: Tracking, Local Mapping and Loop Closing}

Our system, see an overview in Fig. 1, incorporates three threads that run in parallel: tracking, local mapping and loop 
closing. The tracking is in charge of localizing the camera with every frame and deciding when to insert a new keyframe. We perform first an initial feature matching with the previous frame and optimize the pose using motion-only BA. If the tracking is lost (e.g. due to occlusions or abrupt movements), the place recognition module is used to perform a global relocalization. Once there is an initial estimation of the camera pose and feature matchings, a local visible map is retrieved using the covisibility graph of keyframes that is maintained by the system, see Fig. 2(a) and Fig. 2(b). Then matches with the local map points are searched by reprojection, and camera pose is optimized again with all matches. Finally the tracking thread decides if a new keyframe is inserted. All the tracking steps are explained in detail in Section V. The novel procedure to create an initial map is presented in Section IV.

The local mapping processes new keyframes and performs local $B A$ to achieve an optimal reconstruction in the surroundings of the camera pose. New correspondences for unmatched ORB in the new keyframe are searched in connected keyframes in the covisibility graph to triangulate new points. Some time after creation, based on the information gathered during the tracking, an exigent point culling policy is applied in order to retain only high quality points. The local mapping is also in charge of culling redundant keyframes. We explain in detail all local mapping steps in Section VI.

The loop closing searches for loops with every new keyframe. If a loop is detected, we compute a similarity transformation that informs about the drift accumulated in the loop. Then both sides of the loop are aligned and duplicated points are fused. Finally a pose graph optimization over similarity constraints [6] is performed to achieve global consistency. The main novelty is that we perform the optimization over the Essential Graph, a sparser subgraph of the covisibility graph which is explained in Section III-D. The loop detection and correction steps are explained in detail in Section VII.

We use the Levenberg-Marquardt algorithm implemented in g2o [37] to carry out all optimizations. In the Appendix we describe the error terms, cost functions, and variables involved in each optimization.

\section{Map Points, KeyFrames and their Selection}

Each map point $p_{i}$ stores:

- Its 3D position $\mathbf{X}_{w, i}$ in the world coordinate system.

- The viewing direction $\mathbf{n}_{i}$, which is the mean unit vector of all its viewing directions (the rays that join the point with the optical center of the keyframes that observe it).

- A representative ORB descriptor $\mathbf{D}_{i}$, which is the associated ORB descriptor whose hamming distance is minimum with respect to all other associated descriptors in the keyframes in which the point is observed.

- The maximum $d_{\max }$ and minimum $d_{\min }$ distances at which the point can be observed, according to the scale invariance limits of the ORB features.

Each keyframe $K_{i}$ stores:

- The camera pose $\mathbf{T}_{i w}$, which is a rigid body transformation that transforms points from the world to the camera coordinate system.

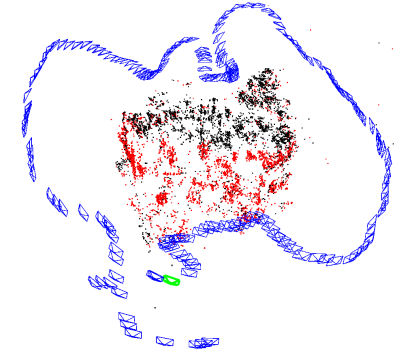

(a) KeyFrames (blue), Current Camera (green), MapPoints (black, red), Current Local MapPoints (red)

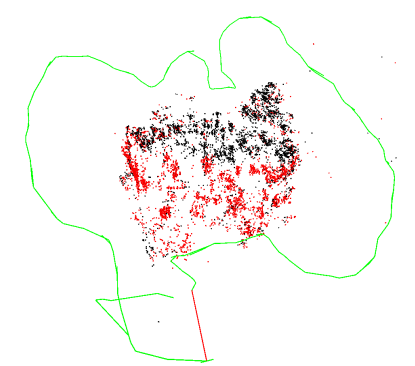

(c) Spanning Tree (green) and Loop Closure (red)

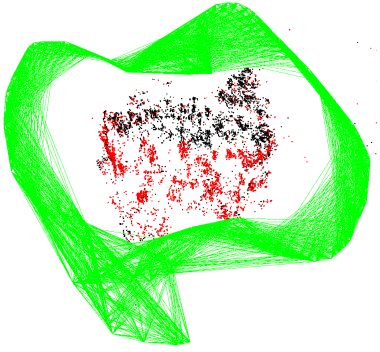

(b) Covisibility Graph
Fig. 2. Reconstruction and graphs in the sequence fr3_long_office_household from the TUM RGB-D Benchmark [38].

- The camera intrinsics, including focal length and principal point.

- All the ORB features extracted in the frame, associated or not to a map point, whose coordinates are undistorted if a distortion model is provided.

Map points and keyframes are created with a generous policy, while a later very exigent culling mechanism is in charge of detecting redundant keyframes and wrongly matched or not trackable map points. This permits a flexible map expansion during exploration, which boost tracking robustness under hard conditions (e.g. rotations, fast movements), while its size is bounded in continual revisits to the same environment, i.e. lifelong operation. Additionally our maps contain very few outliers compared with PTAM, at the expense of containing less points. Culling procedures of map points and keyframes are explained in Sections VI-B and VI-E respectively.

\section{Covisibility Graph and Essential Graph}

Covisibility information between keyframes is very useful in several tasks of our system, and is represented as an undirected weighted graph as in [7]. Each node is a keyframe and an edge between two keyframes exists if they share observations of the same map points (at least 15), being the weight $\theta$ of the edge the number of common map points.

In order to correct a loop we perform a pose graph optimization [6] that distributes the loop closing error along the graph. In order not to include all the edges provided by the covisibility graph, which can be very dense, we propose to build an Essential Graph that retains all the nodes (keyframes), but less edges, still preserving a strong network that yields 
accurate results. The system builds incrementally a spanning tree from the initial keyframe, which provides a connected subgraph of the covisibility graph with minimal number of edges. When a new keyframe is inserted, it is included in the tree linked to the keyframe which shares most point observations, and when a keyframe is erased by the culling policy, the system updates the links affected by that keyframe. The Essential Graph contains the spanning tree, the subset of edges from the covisibility graph with high covisibility $\left(\theta_{\min }=100\right)$, and the loop closure edges, resulting in a strong network of cameras. Fig. 2 shows an example of a covisibility graph, spanning tree and associated essential graph. As shown in the experiments of Section VIII-E, when performing the pose graph optimization, the solution is so accurate that an additional full bundle adjustment optimization barely improves the solution. The efficiency of the essential graph and the influence of the $\theta_{\min }$ is shown at the end of Section VIII-E.

\section{E. Bags of Words Place Recognition}

The system has embedded a bags of words place recognition module, based on DBoW2 $2^{2}$ [5], to perform loop detection and relocalization. Visual words are just a discretization of the descriptor space, which is known as the visual vocabulary. The vocabulary is created offline with the ORB descriptors extracted from a large set of images. If the images are general enough, the same vocabulary can be used for different environments getting a good performance, as shown in our previous work [11]. The system builds incrementally a database that contains an invert index, which stores for each visual word in the vocabulary, in which keyframes it has been seen, so that querying the database can be done very efficiently. The database is also updated when a keyframe is deleted by the culling procedure.

Because there exists visual overlap between keyframes, when querying the database there will not exist a unique keyframe with a high score. The original DBoW2 took this overlapping into account, adding up the score of images that are close in time. This has the limitation of not including keyframes viewing the same place but inserted at a different time. Instead we group those keyframes that are connected in the covisibility graph. In addition our database returns all keyframe matches whose scores are higher than the $75 \%$ of the best score.

An additional benefit of the bags of words representation for feature matching was reported in [5]. When we want to compute the correspondences between two sets of ORB features, we can constraint the brute force matching only to those features that belong to the same node in the vocabulary tree at a certain level (we select the second out of six), speeding up the search. We use this trick when searching matches for triangulating new points, and at loop detection and relocalization. We also refine the correspondences with an orientation consistency test, see [11] for details, that discards outliers ensuring a coherent rotation for all correspondences.

${ }^{2}$ https://github.com/dorian3d/DBoW2

\section{Automatic Map Initialization}

The goal of the map initialization is to compute the relative pose between two frames to triangulate an initial set of map points. This method should be independent of the scene (planar or general) and should not require human intervention to select a good two-view configuration, i.e. a configuration with significant parallax. We propose to compute in parallel two geometrical models, a homography assuming a planar scene and a fundamental matrix assuming a non-planar scene. We then use a heuristic to select a model and try to recover the relative pose with a specific method for the selected model. Our method only initializes when it is certain that the twoview configuration is safe, detecting low-parallax cases and the well-known twofold planar ambiguity [27], avoiding to initialize a corrupted map. The steps of our algorithm are:

1) Find initial correspondences:

Extract ORB features (only at the finest scale) in the current frame $F_{c}$ and search for matches $\mathbf{x}_{c} \leftrightarrow \mathbf{x}_{r}$ in the reference frame $F_{r}$. If not enough matches are found, reset the reference frame.

2) Parallel computation of the two models:

Compute in parallel threads a homography $\mathbf{H}_{c r}$ and a fundamental matrix $\mathbf{F}_{c r}$ :

$$
\mathbf{x}_{c}=\mathbf{H}_{c r} \mathbf{x}_{r} \quad \mathbf{x}_{c}^{T} \mathbf{F}_{c r} \mathbf{x}_{r}=0
$$

with the normalized DLT and 8-point algorithms respectively as explained in [2] inside a RANSAC scheme. To make homogeneous the procedure for both models, the number of iterations is prefixed and the same for both models, along with the points to be used at each iteration, 8 for the fundamental matrix, and 4 of them for the homography. At each iteration we compute a score $S_{M}$ for each model $M$ ( $H$ for the homography, $F$ for the fundamental matrix):

$$
\begin{gathered}
S_{M}=\sum_{i}\left(\rho_{M}\left(d_{c r}^{2}\left(\mathbf{x}_{c}^{i}, \mathbf{x}_{r}^{i}, M\right)\right)+\rho_{M}\left(d_{r c}^{2}\left(\mathbf{x}_{c}^{i}, \mathbf{x}_{r}^{i}, M\right)\right)\right) \\
\rho_{M}\left(d^{2}\right)=\left\{\begin{array}{lll}
\Gamma-d^{2} & \text { if } & d^{2}<T_{M} \\
0 & \text { if } & d^{2} \geq T_{M}
\end{array}\right.
\end{gathered}
$$

where $d_{c r}^{2}$ and $d_{r c}^{2}$ are the symmetric transfer errors [2] from one frame to the other. $T_{M}$ is the outlier rejection threshold based on the $\chi^{2}$ test at $95 \%\left(T_{H}=5.99\right.$, $T_{F}=3.84$, assuming a standard deviation of 1 pixel in the measurement error). $\Gamma$ is defined equal to $T_{H}$ so that both models score equally for the same $d$ in their inlier region, again to make the process homogeneous.

We keep the homography and fundamental matrix with highest score. If no model could be found (not enough inliers), we restart the process again from step 1.

3) Model selection:

If the scene is planar, nearly planar or there is low parallax, it can be explained by a homography. However a fundamental matrix can also be found, but the problem is not well constrained [2] and any attempt to recover the motion from the fundamental matrix would yield 
wrong results. We should select the homography as the reconstruction method will correctly initialize from a plane or it will detect the low parallax case and refuse the initialization. On the other hand a non-planar scene with enough parallax can only be explained by the fundamental matrix, but a homography can also be found explaining a subset of the matches if they lie on a plane or they have low parallax (they are far away). In this case we should select the fundamental matrix. We have found that a robust heuristic is to compute:

$$
R_{H}=\frac{S_{H}}{S_{H}+S_{F}}
$$

and select the homography if $R_{H}>0.45$, which adequately captures the planar and low parallax cases. Otherwise, we select the fundamental matrix.

4) Motion and Structure from Motion recovery:

Once a model is selected we retrieve the motion hypotheses associated. In the case of the homography we retrieve 8 motion hypotheses using the method of Faugeras et. al [23]. The method proposes cheriality tests to select the valid solution. However these tests fail if there is low parallax as points easily go in front or back of the cameras, which could yield the selection of a wrong solution. We propose to directly triangulate the eight solutions, and check if there is one solution with most points seen with parallax, in front of both cameras and with low reprojection error. If there is not a clear winner solution, we do not initialize and continue from step 1. This technique to disambiguate the solutions makes our initialization robust under low parallax and the twofold ambiguity configuration, and could be considered the key of the robustness of our method.

In the case of the fundamental matrix, we convert it in an essential matrix using the calibration matrix $\mathbf{K}$ :

$$
\mathbf{E}_{r c}=\mathbf{K}^{T} \mathbf{F}_{r c} \mathbf{K}
$$

and then retrieve 4 motion hypotheses with the singular value decomposition method explained in [2]. We triangulate the four solutions and select the reconstruction as done for the homography.

5) Bundle adjustment:

Finally we perform a full $B A$, see the Appendix for details, to refine the initial reconstruction.

An example of a challenging initialization in the outdoor NewCollege robot sequence [39] is shown in Fig. 3. It can be seen how PTAM and LSD-SLAM have initialized all points in a plane, while our method has waited until there is enough parallax, initializing correctly from the fundamental matrix.

\section{TRACKING}

In this section we describe the steps of the tracking thread that are performed with every frame from the camera. The camera pose optimizations, mentioned in several steps, consist in motion-only BA, which is described in the Appendix.

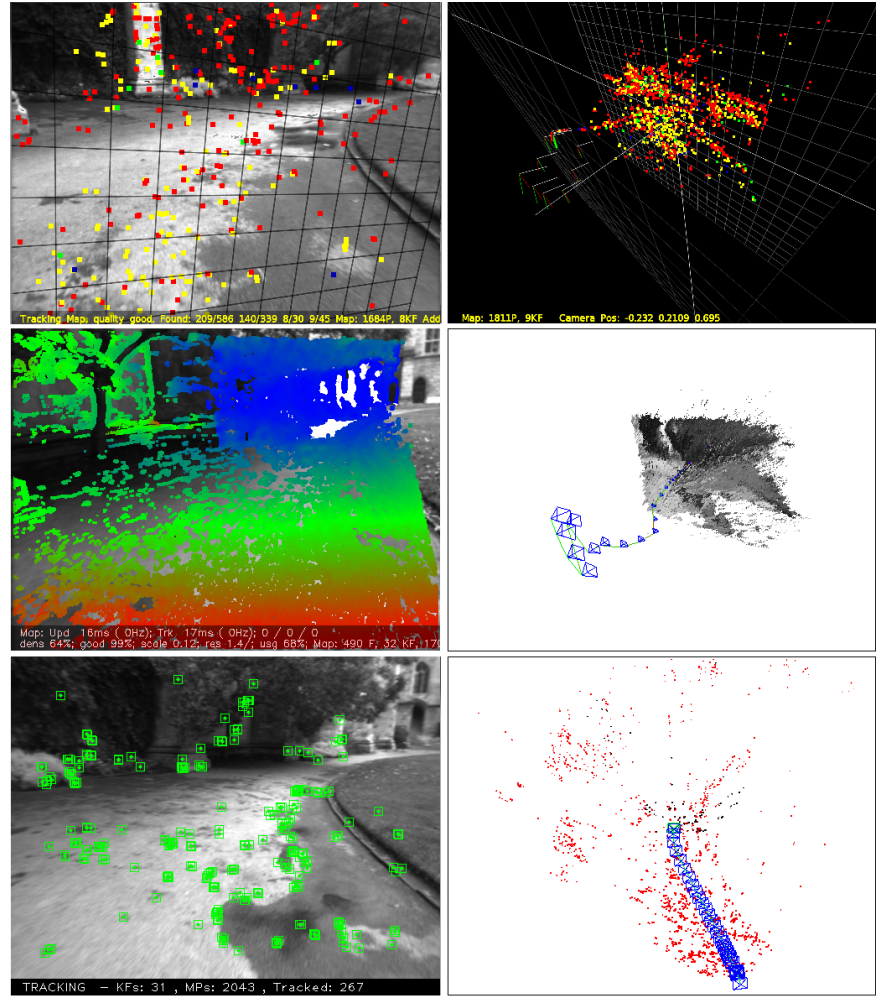

Fig. 3. Top: PTAM, middle LSD-SLAM, bottom: ORB-SLAM, some time after initialization in the NewCollege sequence [39]. PTAM and LSD-SLAM initialize a corrupted planar solution while our method has automatically initialized from the fundamental matrix when it has detected enough parallax. Depending on which keyframes are manually selected, PTAM is also able to initialize well.

\section{A. ORB Extraction}

We extract FAST corners at 8 scale levels with a scale factor of 1.2. For image resolutions from $512 \times 384$ to $752 \times 480$ pixels we found suitable to extract 1000 corners, for higher resolutions, as the $1241 \times 376$ in the KITTI dataset [40] we extract 2000 corners. In order to ensure an homogeneous distribution we divide each scale level in a grid, trying to extract at least 5 corners per cell. Then we detect corners in each cell, adapting the detector threshold if not enough corners are found. The amount of corners retained per cell is also adapted if some cells contains no corners (textureless or low contrast). The orientation and ORB descriptor are then computed on the retained FAST corners. The ORB descriptor is used in all feature matching, in contrast to the search by patch correlation in PTAM.

\section{B. Initial Pose Estimation from Previous Frame}

If tracking was successful for last frame, we use a constant velocity motion model to predict the camera pose and perform a guided search of the map points observed in the last frame. If not enough matches were found (i.e. motion model is clearly violated), we use a wider search of the map points around their position in the last frame. The pose is then optimized with the found correspondences. 


\section{Initial Pose Estimation via Global Relocalization}

If the tracking is lost, we convert the frame into bag of words and query the recognition database for keyframe candidates for global relocalization. We compute correspondences with ORB associated to map points in each keyframe, as explained in section III-E. We then perform alternatively RANSAC iterations for each keyframe and try to find a camera pose using the $\mathrm{PnP}$ algorithm [41]. If we find a camera pose with enough inliers, we optimize the pose and perform a guided search of more matches with the map points of the candidate keyframe. Finally the camera pose is again optimized, and if supported with enough inliers, tracking procedure continues.

\section{Track Local Map}

Once we have an estimation of the camera pose and an initial set of feature matches, we can project the map into the frame and search more map point correspondences. To bound the complexity in large maps, we only project a local map. This local map contains the set of keyframes $\mathcal{K}_{1}$, that share map points with the current frame, and a set $\mathcal{K}_{2}$ with neighbors to the keyframes $\mathcal{K}_{1}$ in the covisibility graph. The local map also has a reference keyframe $K_{\text {ref }} \in \mathcal{K}_{1}$ which shares most map points with current frame. Now each map point seen in $\mathcal{K}_{1}$ and $\mathcal{K}_{2}$ is searched in the current frame as follows:

1) Compute the map point projection $\mathrm{x}$ in the current frame. Discard if it lays out of the image bounds.

2) Compute the angle between the current viewing ray $\mathbf{v}$ and the map point mean viewing direction $\mathbf{n}$. Discard if $\mathbf{v} \cdot \mathbf{n}<\cos \left(60^{\circ}\right)$.

3) Compute the distance $d$ from map point to camera center. Discard if it is out of the scale invariance region of the map point $d \notin\left[d_{\min }, d_{\max }\right]$.

4) Compute the scale in the frame by the ratio $d / d_{\min }$.

5) Compare the representative descriptor $\mathbf{D}$ of the map point with the still unmatched ORB features in the frame, at the predicted scale, and near $\mathbf{x}$, and associate the map point with the best match.

The camera pose is finally optimized with all the map points found in the frame.

\section{E. New Keyframe Decision}

The last step is to decide if the current frame is spawned as a new keyframe. As there is a mechanism in the local mapping to cull redundant keyframes, we will try to insert keyframes as fast as possible, because that makes the tracking more robust to challenging camera movements, typically rotations. To insert a new keyframe all the following conditions must be met:

1) More than 20 frames must have passed from the last global relocalization.

2) Local mapping is idle, or more than 20 frames have passed from last keyframe insertion.

3) Current frame tracks at least 50 points.

4) Current frame tracks less than $90 \%$ points than $K_{\text {ref }}$.

Instead of using a distance criterion to other keyframes as PTAM, we impose a minimum visual change (condition
4). Condition 1 ensures a good relocalization and condition 3 a good tracking. If a keyframe is inserted when the local mapping is busy (second part of condition 2), a signal is sent to stop local bundle adjustment, so that it can process as soon as possible the new keyframe.

\section{LOCAL MAPPING}

In this section we describe the steps performed by the local mapping with every new keyframe $K_{i}$.

\section{A. KeyFrame Insertion}

At first we update the covisibility graph, adding a new node for $K_{i}$ and updating the edges resulting from the shared map points with other keyframes. We then update the spanning tree linking $K_{i}$ with the keyframe with most points in common. We then compute the bags of words representation of the keyframe, that will help in the data association for triangulating new points.

\section{B. Recent Map Points Culling}

Map points, in order to be retained in the map, must pass a restrictive test during the first three keyframes after creation, that ensures that they are trackable and not wrongly triangulated, i.e due to spurious data association. A point must fulfill these two conditions:

1) The tracking must find the point in more than the $25 \%$ of the frames in which it is predicted to be visible.

2) If more than one keyframe has passed from map point creation, it must be observed from at least three keyframes.

Once a map point have passed this test, it can only be removed if at any time it is observed from less than three keyframes. This can happen when keyframes are culled and when local bundle adjustment discards outlier observations. This policy makes our map contain very few outliers.

\section{New Map Point Creation}

New map points are created by triangulating ORB from connected keyframes $\mathcal{K}_{c}$ in the covisibility graph. For each unmatched ORB in $K_{i}$ we search a match with other unmatched point in other keyframe. This matching is done as explained in Section III-E and discard those matches that do not fulfill the epipolar constraint. ORB pairs are triangulated, and to accept the new points, positive depth in both cameras, parallax, reprojection error and scale consistency are checked. Initially a map point is observed from two keyframes but it could be matched in others, so it is projected in the rest of connected keyframes, and correspondences are searched as detailed in section V-D.

\section{Local Bundle Adjustment}

The local $B A$ optimizes the currently processed keyframe $K_{i}$, all the keyframes connected to it in the covisibility graph $\mathcal{K}_{c}$, and all the map points seen by those keyframes. All other keyframes that see those points but are not connected to the 
currently processed keyframe are included in the optimization but remain fixed. Observations that are marked as outliers are discarded at the middle and at the end of the optimization. See the Appendix for more details about this optimization.

\section{E. Local Keyframe Culling}

In order to maintain a compact reconstruction, the local mapping tries to detect redundant keyframes and delete them. This is beneficial as bundle adjustment complexity grows with the number of keyframes, but also because it enables lifelong operation in the same environment as the number of keyframes will not grow unbounded, unless the visual content in the scene changes. We discard all the keyframes in $\mathcal{K}_{c}$ whose $90 \%$ of the map points have been seen in at least other three keyframes in the same or finer scale. The scale condition ensures that map points maintain keyframes from which they are measured with most accuracy. This policy was inspired by the one proposed in the work of Tan et. al [24], where keyframes were discarded after a process of change detection.

\section{LOOP CLOSING}

The loop closing thread takes $K_{i}$, the last keyframe processed by the local mapping, and tries to detect and close loops. The steps are next described.

\section{A. Loop Candidates Detection}

At first we compute the similarity between the bag of words vector of $K_{i}$ and all its neighbors in the covisibility graph $\left(\theta_{\min }=30\right)$ and retain the lowest score $s_{\min }$. Then we query the recognition database and discard all those keyframes whose score is lower than $s_{\min }$. This is a similar operation to gain robustness as the normalizing score in DBoW2, which is computed from the previous image, but here we use covisibility information. In addition all those keyframes directly connected to $K_{i}$ are discarded from the results. To accept a loop candidate we must detect consecutively three loop candidates that are consistent (keyframes connected in the covisibility graph). There can be several loop candidates if there are several places with similar appearance to $K_{i}$.

\section{B. Compute the Similarity Transformation}

In monocular SLAM there are seven degrees of freedom in which the map can drift, three translations, three rotations and a scale factor [6]. Therefore to close a loop we need to compute a similarity transformation from the current keyframe $K_{i}$ to the loop keyframe $K_{l}$ that informs us about the error accumulated in the loop. The computation of this similarity will serve also as geometrical validation of the loop.

We first compute correspondences between ORB associated to map points in the current keyframe and the loop candidate keyframes, following the procedure explained in section III-E. At this point we have $3 \mathrm{D}$ to $3 \mathrm{D}$ correspondences for each loop candidate. We alternatively perform RANSAC iterations with each candidate, trying to find a similarity transformation using the method of Horn [42]. If we find a similarity $\mathbf{S}_{i l}$ with enough inliers, we optimize it (see the Appendix), and perform a guided search of more correspondences. We optimize it again and, if $\mathbf{S}_{i l}$ is supported by enough inliers, the loop with $K_{l}$ is accepted.

\section{Loop Fusion}

The first step in the loop correction is to fuse duplicated map points and insert new edges in the covisibility graph that will attach the loop closure. At first the current keyframe pose $\mathbf{T}_{i w}$ is corrected with the similarity transformation $\mathbf{S}_{i l}$ and this correction is propagated to all the neighbors of $K_{i}$, concatenating transformations, so that both sides of the loop get aligned. All map points seen by the loop keyframe and its neighbors are projected into $K_{i}$ and its neighbors and matches are searched in a narrow area around the projection, as done in section V-D. All those map points matched and those that were inliers in the computation of $\mathbf{S}_{i l}$ are fused. All keyframes involved in the fusion will update their edges in the covisibility graph effectively creating edges that attach the loop closure.

\section{Essential Graph Optimization}

To effectively close the loop, we perform a pose graph optimization over the Essential Graph, described in Section III-D, that distributes the loop closing error along the graph. The optimization is performed over similarity transformations to correct the scale drift [6]. The error terms and cost function are detailed in the Appendix. After the optimization each map point is transformed according to the correction of one of the keyframes that observes it.

\section{EXPERIMENTS}

We have performed an extensive experimental validation of our system in the large robot sequence of NewCollege [39], evaluating the general performance of the system, in 16 handheld indoor sequences of the TUM RGB-D benchmark [38], evaluating the localization accuracy, relocalization and lifelong capabilities, and in 10 car outdoor sequences from the KITTI dataset [40], evaluating real-time large scale operation, localization accuracy and efficiency of the pose graph optimization.

Our system runs in real time and processes the images exactly at the frame rate they were acquired. We have carried out all experiments with an Intel Core i7-4700MQ (4 cores @ 2.40GHz) and 8Gb RAM. ORB-SLAM has three main threads, that run in parallel with other tasks from ROS and the operating system, which introduces some randomness in the results. For this reason, in some experiments, we report the median from several runs.

\section{A. System Performance in the NewCollege Dataset}

The NewCollege dataset [39] contains a $2.2 \mathrm{~km}$ sequence from a robot traversing a campus and adjacent parks. The sequence is recorded by a stereo camera at $20 \mathrm{fps}$ and a resolution $512 \times 382$. It contains several loops and fast rotations that makes the sequence quite challenging for monocular vision. To the best of our knowledge there is no other monocular system in the literature able to process this whole sequence. For example Strasdat et al. [7], despite being able to close 
TABLE II

LOOP CLOSING TIMES IN NEWCOLLEGE

\begin{tabular}{|c|c|c|c|c|c|c|c|}
\cline { 3 - 7 } \multicolumn{2}{c}{} & \multicolumn{2}{c|}{ Loop Detection (ms) } & \multicolumn{2}{c|}{ Loop Correction (s) } & \\
\hline Loop & KeyFrames & $\begin{array}{c}\text { Essential Graph } \\
\text { Edges }\end{array}$ & $\begin{array}{c}\text { Candidates } \\
\text { Detection }\end{array}$ & $\begin{array}{c}\text { Similarity } \\
\text { Transformation }\end{array}$ & Fusion & $\begin{array}{c}\text { Essential Graph } \\
\text { Optimization }\end{array}$ & Total (s) \\
\hline 1 & 287 & 1347 & 4.71 & 20.77 & 0.20 & 0.26 & 0.51 \\
\hline 2 & 1082 & 5950 & 4.14 & 17.98 & 0.39 & 1.06 & 1.52 \\
\hline 3 & 1279 & 7128 & 9.82 & 31.29 & 0.95 & 1.26 & 2.27 \\
\hline 4 & 2648 & 12547 & 12.37 & 30.36 & 0.97 & 2.30 & 3.33 \\
\hline 5 & 3150 & 16033 & 14.71 & 41.28 & 1.73 & 2.80 & 4.60 \\
\hline 6 & 4496 & 21797 & 13.52 & 48.68 & 0.97 & 3.62 & 4.69 \\
\hline
\end{tabular}

TABLE I

Tracking AND MapPing times in NewCollege

\begin{tabular}{|c|l|c|c|c|}
\hline Thread & Operation & $\begin{array}{c}\text { Median } \\
(\mathrm{ms})\end{array}$ & $\begin{array}{c}\text { Mean } \\
(\mathrm{ms})\end{array}$ & $\begin{array}{c}\text { Std } \\
(\mathrm{ms})\end{array}$ \\
\hline \hline \multirow{4}{*}{ TRACKING } & ORB extraction & 11.10 & 11.42 & 1.61 \\
\cline { 2 - 5 } & Initial Pose Est. & 3.38 & 3.45 & 0.99 \\
\cline { 2 - 5 } & Track Local Map & 14.84 & 16.01 & 9.98 \\
\cline { 2 - 5 } & Total & 30.57 & 31.60 & 10.39 \\
\hline \hline \multirow{3}{*}{\begin{tabular}{l} 
LACAL \\
\cline { 2 - 5 }
\end{tabular}} & KeyFrame Insertion & 10.29 & 11.88 & 5.03 \\
\cline { 2 - 5 } & Map Point Culling & 0.10 & 3.18 & 6.70 \\
\cline { 2 - 5 } & Map Point Creation & 66.79 & 72.96 & 31.48 \\
\cline { 2 - 5 } & Local BA & 296.08 & 360.41 & 171.11 \\
\cline { 2 - 5 } & KeyFrame Culling & 8.07 & 15.79 & 18.98 \\
\cline { 2 - 5 } & Total & 383.59 & 464.27 & 217.89 \\
\hline
\end{tabular}

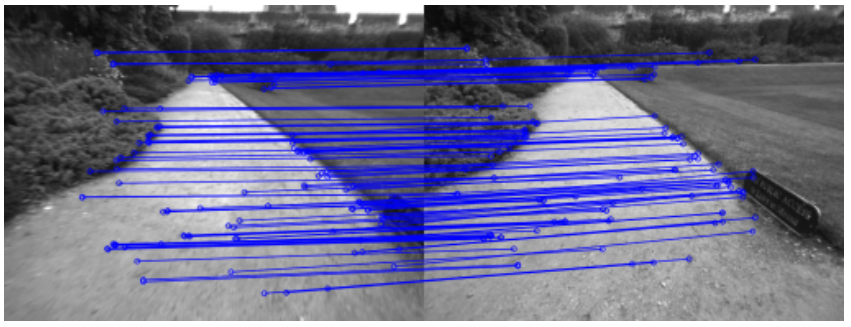

Fig. 4. Example of loop detected in the NewCollege sequence. We draw the inlier correspondences supporting the similarity transformation found.

loops and work in large scale environments, only showed monocular results for a small part of this sequence.

As an example of our loop closing procedure we show in Fig. 4 the detection of a loop with the inliers that support the similarity transformation. Fig. 5 shows the reconstruction before and after the loop closure. In red it is shown the local map, which after the loop closure extends along both sides of the loop closure. The whole map after processing the full sequence at its real frame-rate is shown in Fig. 6. The big loop on the right does not perfectly align because it was traversed in opposite directions and the place recognizer was not able to find loop closures.

We have extracted statistics of the times spent by each thread in this experiment. Table I shows the results for the
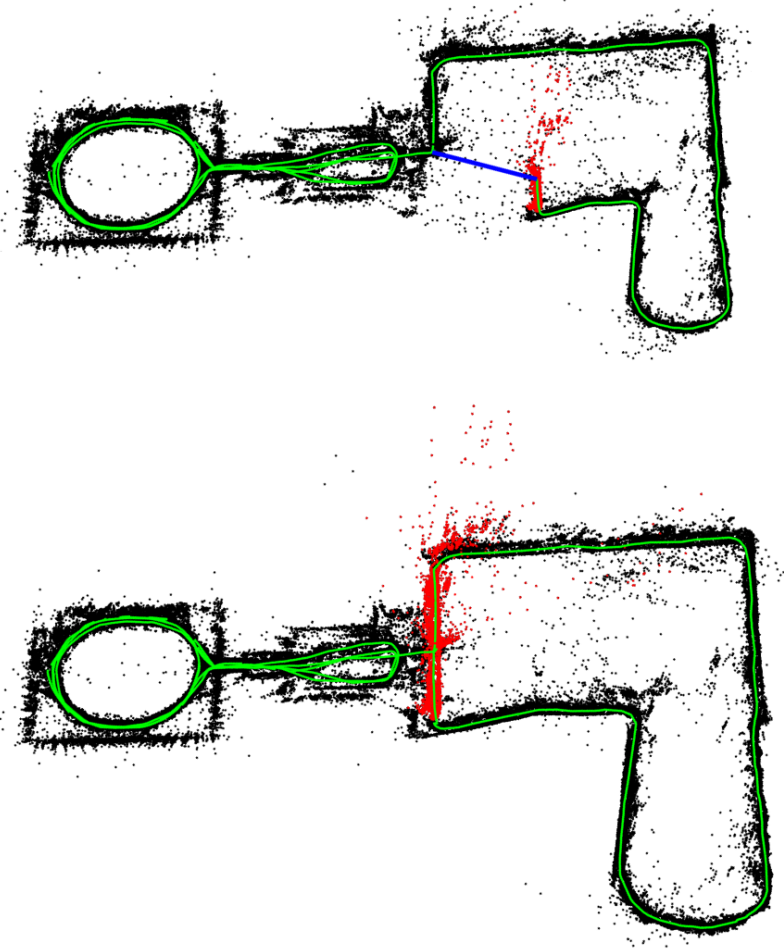

Fig. 5. Map before and after a loop closure in the NewCollege sequence. The loop closure match is drawn in blue, the trajectory in green, and the local map for the tracking at that moment in red. The local map is extended along both sides of the loop after it is closed.

tracking and the local mapping. Tracking works at frame-rates around $25-30 \mathrm{~Hz}$, being the most demanding task to track the local map. If needed this time could be reduced limiting the number of keyframes that are included in the local map. In the local mapping thread the most demanding task is local bundle adjustment. The local BA time varies if the robot is exploring or in a well mapped area, because during exploration bundle adjustment is interrupted if tracking inserts a new keyframe, as explained in section V-E. In case of not needing new keyframes local bundle adjustment performs a generous number of prefixed iterations.

Table II shows the results for each of the 6 loop closures found. It can be seen how the loop detection increases sublinearly with the number of keyframes. This is due to 


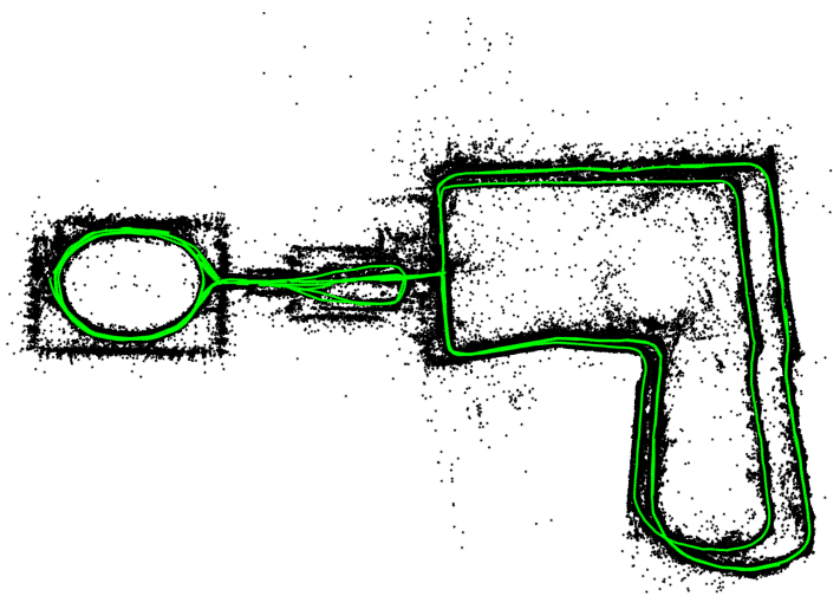

Fig. 6. ORB-SLAM reconstruction of the full sequence of NewCollege. The bigger loop on the right is traversed in opposite directions and not visual loop closures were found, therefore they do not perfectly align.

the efficient querying of the database that only compare the subset of images with words in common, which demonstrates the potential of bag of words for place recognition. Our Essential Graph includes edges around 5 times the number of keyframes, which is a quite sparse graph.

\section{B. Localization Accuracy in the TUM RGB-D Benchmark}

The TUM RGB-D benchmark [38] is an excellent dataset to evaluate the accuracy of camera localization as it provides several sequences with accurate ground truth obtained with an external motion capture system. We have discarded all those sequences that we consider that are not suitable for pure monocular SLAM systems, as they contain strong rotations, no texture or no motion.

For comparison we have also executed the novel, direct, semi-dense LSD-SLAM [10] and PTAM [4] in the benchmark. We compare also with the trajectories generated by RGBDSLAM [43] which are provided for some of the sequences in the benchmark website. In order to compare ORB-SLAM, LSD-SLAM and PTAM with the ground truth, we align the keyframe trajectories using a similarity transformation, as scale is unknown, and measure the absolute trajectory error (ATE) [38]. In the case of RGBD-SLAM we align the trajectories with a rigid body transformation, but also a similarity to check if the scale was well recovered. LSD-SLAM initializes from random depth values and takes time to converge, therefore we have discarded the first 10 keyframes when comparing with the ground truth. For PTAM we manually selected two frames from which we get a good initialization. Table III shows the median results over 5 executions in each of the 16 sequences selected.

It can be seen that ORB-SLAM is able to process all the sequences, except for fr3_nostructure_texture_far (fr3_nstr_tex_far). This is a planar scene that because of the camera trajectory with respect to the plane has two possible interpretations, i.e. the twofold ambiguity described in [27]. Our initialization method detects the ambiguity and for safety refuses to initialize. PTAM initializes selecting sometimes the true solution and others the corrupted one, in which case the error is unacceptable. We have not noticed two different reconstructions from LSD-SLAM but the error in this sequence is very high. In the rest of the sequences, PTAM and LSD-SLAM exhibit less robustness than our method, loosing track in eight and three sequences respectively.

In terms of accuracy ORB-SLAM and PTAM are similar in open trajectories, while ORB-SLAM achieves higher accuracy when detecting large loops as in the sequence fr3_nostructure_texture_near_withloop (fr3_nstr_tex_near). The most surprising results is that both PTAM and ORBSLAM are clearly more accurate than LSD-SLAM and RGBD-SLAM. One of the possible causes can be that they reduce the map optimization to a pose-graph optimization were sensor measurements are discarded, while we perform bundle adjustment and jointly optimize cameras and map over sensor measurements, which is the gold standard algorithm to solve structure from motion [2]. We further discuss this result in Section IX-B. Another interesting result is that LSD-SLAM seems to be less robust to dynamic objects than our system as seen in fr2_desk_with_person and fr3_walking_xyz.

We have noticed that RGBD-SLAM has a bias in the scale in $f r 2$ sequences, as aligning the trajectories with $7 \mathrm{DoF}$ significantly reduces the error. Finally it should be noted that Engel et al. [10] reported that PTAM has less accuracy than LSD-SLAM in $f r 2 \_x y z$ with an RMSE of $24.28 \mathrm{~cm}$. However, the paper does not give enough details on how those results were obtained, and we have been unable to reproduce them.

\section{Relocalization in the TUM RGB-D Benchmark}

We perform two relocalization experiments in the TUM RGB-D benchmark. In the first experiment we build a map with the first 30 seconds of the sequence $f r 2 \_x y z$ and perform global relocalization with every successive frame and evaluate the accuracy of the recovered poses. We perform the same experiment with PTAM for comparison. Fig. 7 shows the keyframes used to create the initial map, the poses of the relocalized frames and the ground truth for those frames. It can be seen that PTAM is only able to relocalize frames which are near to the keyframes due to the little invariance of its relocalization method. Table IV shows the recall and the error with respect to the ground truth. ORB-SLAM accurately relocalizes more than the double of frames than PTAM. In the second experiment we create an initial map with sequence fr3_sitting_xyz and try to relocalize all frames from fr3_walking_xyz. This is a challenging experiment as there are big occlusions due to people moving in the scene. Here PTAM finds no relocalizations while our system relocalizes $78 \%$ of the frames, as can be seen in Table IV. Fig. 8 shows some examples of challenging relocalizations performed by our system in these experiments.

\section{Lifelong Experiment in the TUM RGB-D Benchmark}

Previous relocalization experiments have shown that our system is able to localize in a map from very different viewpoints and robustly under moderate dynamic changes. This property in conjunction with our keyframe culling procedure 
TABLE III

KeYFrame LOCALIZATION ERROR COMPARISON IN THE TUM RGB-D BENCHMARK [38]

\begin{tabular}{|c|c|c|c|c|}
\hline & \multicolumn{4}{|c|}{ Absolute KeyFrame Trajectory RMSE (cm) } \\
\hline & ORB-SLAM & PTAM & LSD-SLAM & $\begin{array}{l}\text { RGBD- } \\
\text { SLAM }\end{array}$ \\
\hline fr1_xyz & 0.90 & 1.15 & 9.00 & $1.34(1.34)$ \\
\hline fr2_xyz & 0.30 & 0.20 & 2.15 & $2.61(1.42)$ \\
\hline fr1_floor & 2.99 & $\mathrm{X}$ & 38.07 & $3.51(3.51)$ \\
\hline fr1_desk & 1.69 & $\mathrm{X}$ & 10.65 & $2.58(2.52)$ \\
\hline $\begin{array}{l}\text { fr2_360 } \\
\text { _kidnap }\end{array}$ & 3.81 & 2.63 & $\mathrm{X}$ & $393.3(100.5)$ \\
\hline fr2_desk & 0.88 & $\mathrm{X}$ & 4.57 & $9.50(3.94)$ \\
\hline $\begin{array}{c}\text { fr3_long } \\
\text { _office }\end{array}$ & 3.45 & $\mathrm{X}$ & 38.53 & - \\
\hline $\begin{array}{l}\text { fr3_nstr_ } \\
\text { tex_far }\end{array}$ & $\begin{array}{l}\text { ambiguity } \\
\text { detected }\end{array}$ & $\begin{array}{l}4.92 / \\
34.74\end{array}$ & 18.31 & - \\
\hline $\begin{array}{l}\text { fr3_nstr_ } \\
\text { tex_near }\end{array}$ & 1.39 & 2.74 & 7.54 & - \\
\hline $\begin{array}{l}\text { fr3_str_- } \\
\text { tex_far }\end{array}$ & 0.77 & 0.93 & 7.95 & - \\
\hline $\begin{array}{l}\text { fr3_str_ } \\
\text { tex_near }\end{array}$ & 1.58 & 1.04 & $\mathrm{X}$ & - \\
\hline $\begin{array}{l}\text { fr2_desk } \\
\text { _person }\end{array}$ & 0.63 & $X$ & 31.73 & $6.97(2.00)$ \\
\hline $\begin{array}{c}\text { fr3_sit_ } \\
\text { xyz }\end{array}$ & 0.79 & 0.83 & 7.73 & - \\
\hline $\begin{array}{l}\text { fr3_sit__ } \\
\text { _halfsph }\end{array}$ & 1.34 & $\mathrm{X}$ & 5.87 & - \\
\hline $\begin{array}{c}\text { fr3_walk } \\
\text { _xyz }\end{array}$ & 1.24 & $X$ & 12.44 & - \\
\hline $\begin{array}{l}\text { fr3_walk } \\
\text { _halfsph }\end{array}$ & 1.74 & $\mathrm{X}$ & $\mathrm{X}$ & - \\
\hline
\end{tabular}

Results for ORB-SLAM, PTAM and LSD-SLAM are the median over 5 executions in each sequence. The trajectories have been aligned with 7DoF with the ground truth. Trajectories for RGBD-SLAM are taken from the benchmark website, only available for fr1 and fr2 sequences, and have been aligned with $6 \mathrm{DoF}$ and $7 \mathrm{DoF}$ (results between brackets). $\mathrm{X}$ means that the tracking is lost at some point and a significant portion of the sequence is not processed by the system.

allows to operate lifelong in the same environment under different viewpoints and some dynamic changes.

In the case of a completely static scenario our system is able to maintain the number of keyframes bounded even if the camera is looking at the scene from different viewpoints. We demonstrate it in a custom sequence were the camera is looking at the same desk during 93 seconds but performing a trajectory so that the viewpoint is always changing. We compare the evolution of the number of keyframes in our map and those generated by PTAM in Fig. 9. It can be seen how PTAM is always inserting keyframes, while our mechanism to prune redundant keyframes makes its number to saturate.

While the lifelong operation in a static scenario should be a requirement of any SLAM system, more interesting is the case where dynamic changes occur. We analyze the behavior of our system in such scenario by running consecutively the dynamic sequences from fr3: sitting_xyz, sitting_halfsphere, sitting_rpy,
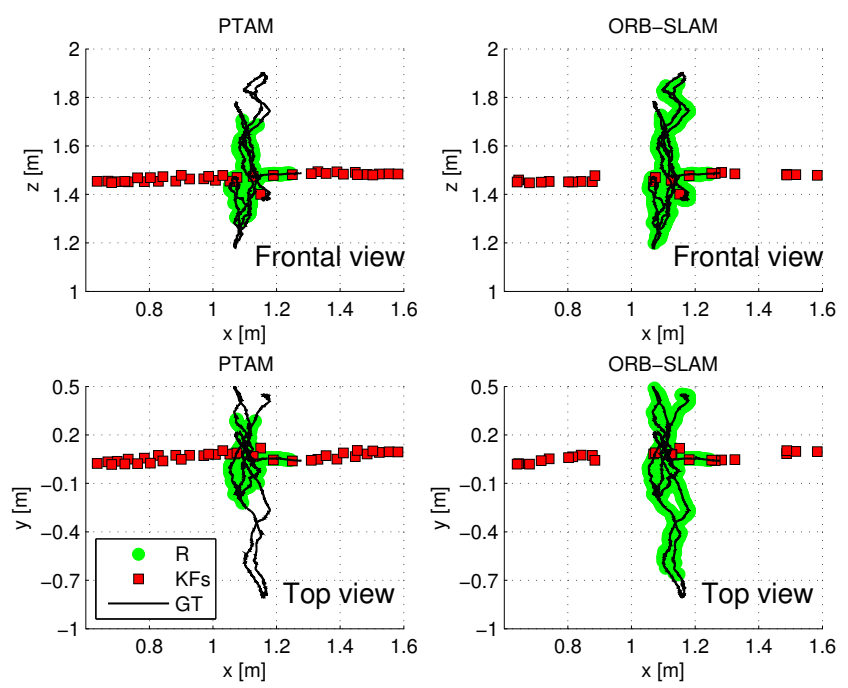

Fig. 7. Relocalization experiment in $f r 2 \_x y z$. Map is initially created during the first 30 seconds of the sequence (KFs). The goal is to relocalize subsequent frames. Successful relocalizations (R) of our system and PTAM are shown. The ground truth (GT) is only shown for the frames to relocalize.
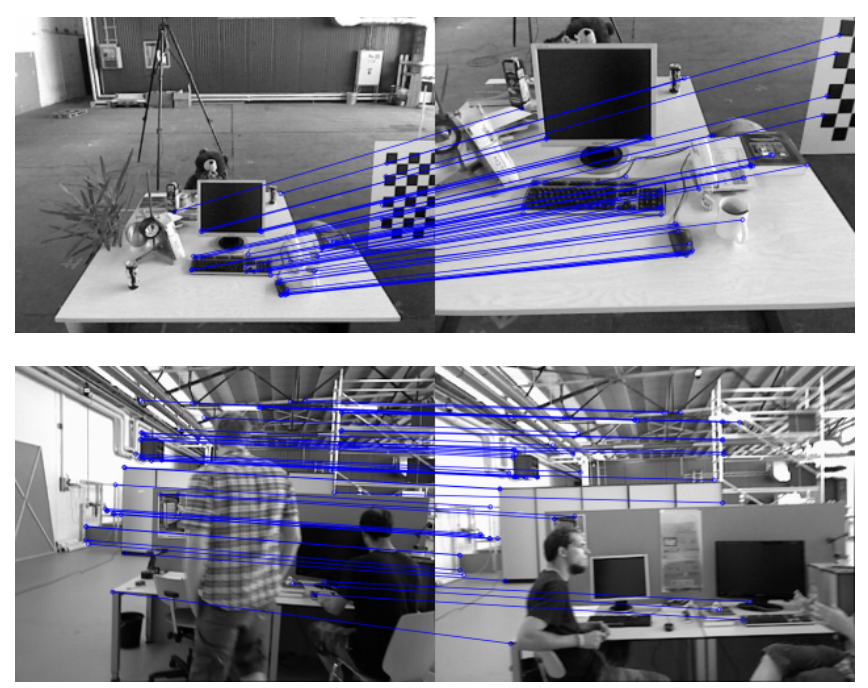

Fig. 8. Example of challenging relocalizations (severe scale change, dynamic objects) that our system successfully found in the relocalization experiments.

walking_xyz, walking_halfspehere and walking_rpy. All the sequences focus the camera to the same desk but perform different trajectories, while people are moving and change some objects like chairs. Fig. 10(a) shows the evolution of the total number of keyframes in the map, and Fig. 10(b) shows for each keyframe its frame of creation and destruction, showing how long the keyframes have survived in the map. It can be seen that during the first two sequences the map size grows as all the views of the scene are being seen for the first time. In Fig. 10(b) we can see that several keyframes created during these two first sequences are maintained in the map during the whole experiment. During the sequences sitting_rpy and walking_xyz the map does not grow, because the map created so far explains well the scene. In contrast, during the last two sequences, more keyframes are inserted showing that there are some novelties in the scene that were not yet represented, 
TABLE IV

RESULTS FOR THE RELOCALIZATION EXPERIMENTS

\begin{tabular}{|c|c|c|c|c|c|}
\cline { 2 - 6 } \multicolumn{1}{c|}{} & Initial Map & \multicolumn{3}{c|}{ Relocalization } \\
\hline System & KFs & $\begin{array}{c}\text { RMSE } \\
(\mathrm{cm})\end{array}$ & $\begin{array}{c}\text { Recall } \\
(\%)\end{array}$ & $\begin{array}{c}\text { RMSE } \\
(\mathrm{cm})\end{array}$ & $\begin{array}{c}\text { Max. Error } \\
(\mathrm{cm})\end{array}$ \\
\hline fr2_xyz. 2769 frames to relocalize \\
\hline PTAM & 37 & 0.19 & 34.9 & 0.26 & 1.52 \\
\hline ORB-SLAM & 24 & 0.19 & $\mathbf{7 8 . 4}$ & 0.38 & 1.67 \\
\hline \multicolumn{7}{|c|}{ fr3_walking_xyz. 859 frames to relocalize } \\
\hline PTAM & 34 & 0.83 & 0.0 & - & - \\
\hline ORB-SLAM & 31 & 0.82 & $\mathbf{7 7 . 9}$ & 1.32 & 4.95 \\
\hline
\end{tabular}

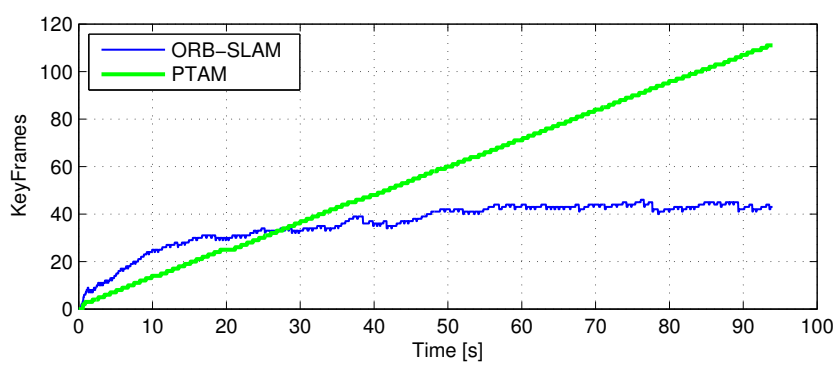

Fig. 9. Lifelong experiment in a static environment where the camera is always looking at the same place from different viewpoints. PTAM is always inserting keyframes, while ORB-SLAM is able to prune redundant keyframes and maintains a bounded-size map.

due probably to dynamic changes. Finally Fig. 10(c) shows a histogram of the keyframes according to the time they have survived with respect to the remaining time of the sequence from its moment of creation. It can be seen that most of the keyframes are destroyed by the culling procedure soon after creation, and only a small subset survive until the end of the experiment. On one hand, this shows that our system has a generous keyframe spawning policy, which is very useful when performing abrupt motions in exploration. On the other hand the system is eventually able to select a small representative subset of those keyframes.

In these lifelong experiments we have shown that our map grows with the content of the scene but not with the time, and that is able to store the dynamic changes of the scene which could be useful to perform some scene understanding by accumulating experience in an environment.

\section{E. Large Scale and Large Loop Closing in the KITTI Dataset}

The odometry benchmark from the KITTI dataset [40] contains 11 sequences from a car driven around a residential area with accurate ground truth from GPS and a Velodyne laser scanner. This is a very challenging dataset for monocular vision due to fast rotations, areas with lot of foliage, which make more difficult data association, and relatively high car speed, being the sequences recorded at $10 \mathrm{fps}$. We play the sequences at the real frame-rate they were recorded and ORBSLAM is able to process all the sequences by the exception of sequence 01 which is a highway with few trackable close objects. Sequences 00, 02, 05, 06, 07, 09 contain loops that

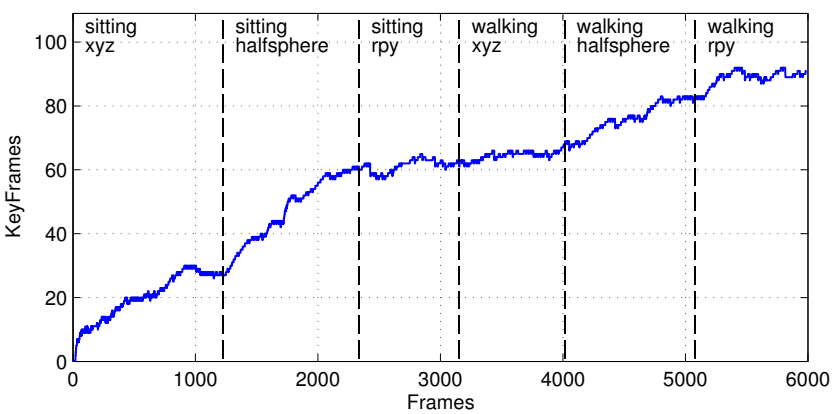

(a) Evolution of the number of keyframes in the map

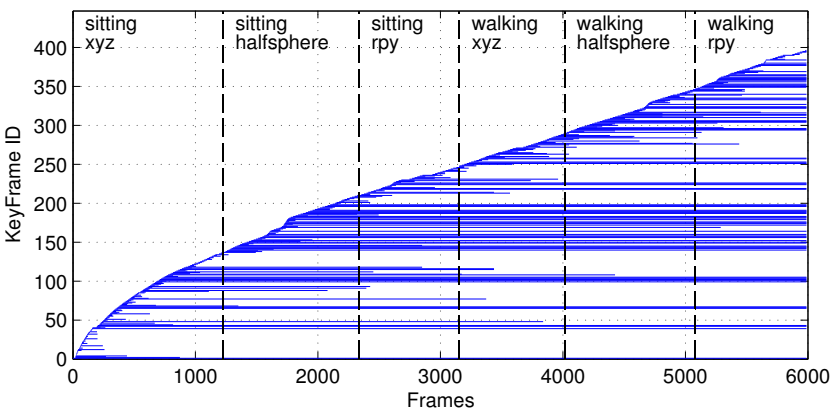

(b) Keyframe creation and destruction. Each horizontal line corresponds to a keyframe, from its creation frame until its destruction

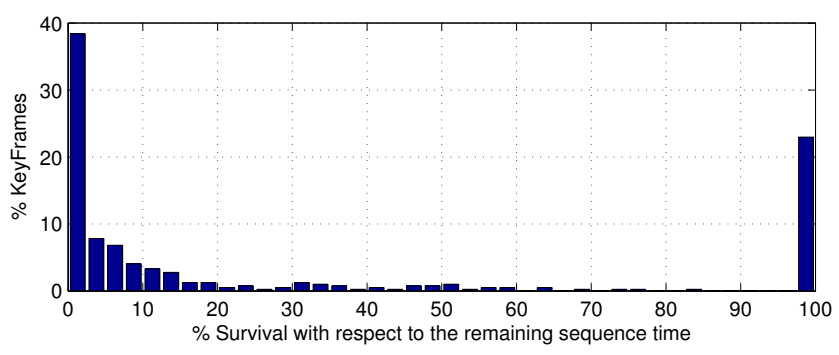

(c) Histogram of the survival time of all spawned keyframes with respect to the remaining time of the experiment

Fig. 10. Lifelong experiment in a dynamic environment from the TUM RGBD Benchmark.

were correctly detected and closed by our system. Sequence 09 contains a loop that can be detected only in a few frames at the end of the sequence, and our system not always detects it (the results provided are for the executions in which it was detected).

Qualitative comparisons of our trajectories and the ground truth are shown in Fig. 11 and Fig. 12. As in the TUM RGB-D benchmark we have aligned the keyframe trajectories of our system and the ground truth with a similarity transformation. We can compare qualitatively our results from Fig. 11 and Fig. 12 with the results provided for sequences 00, 05, 06, 07 and 08 by the recent monocular SLAM approach of Lim et. al [25] in their figure 10. ORB-SLAM produces clearly more accurate trajectories for all those sequences by the exception of sequence 08 in which they seem to suffer less drift.

Table V shows the median RMSE error of the keyframe trajectory over five executions in each sequence. We also provide the dimensions of the maps to put in context the errors. The results demonstrate that our system is very accurate being 

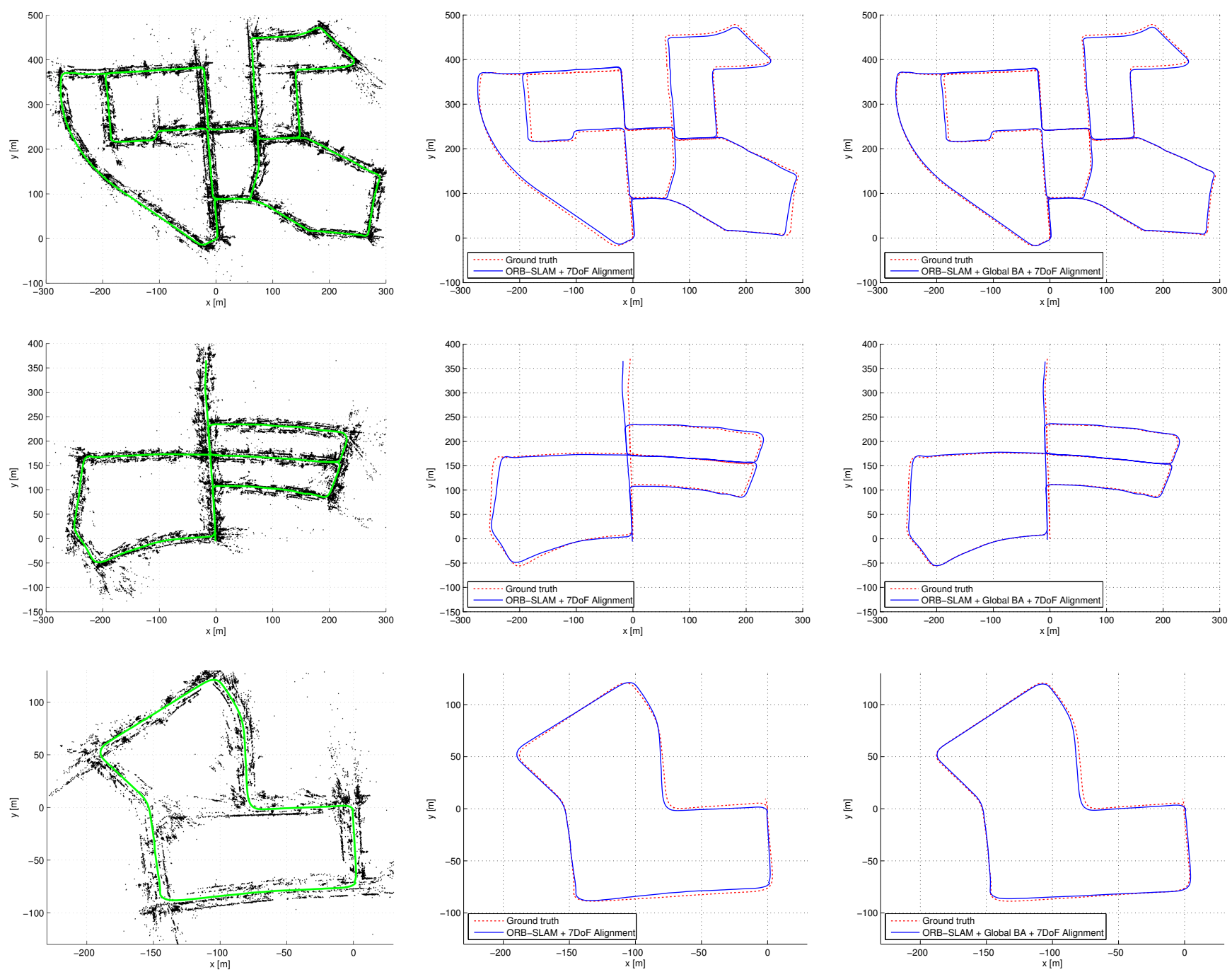

Fig. 11. Sequences 00, 05 and 07 from the odometry benchmark of the KITTI dataset. Left: points and keyframe trajectory. Center: trajectory and ground truth. Right: trajectory after 20 iterations of full BA. The output of our system is quite accurate, while it can be slightly improved with some iterations of BA.

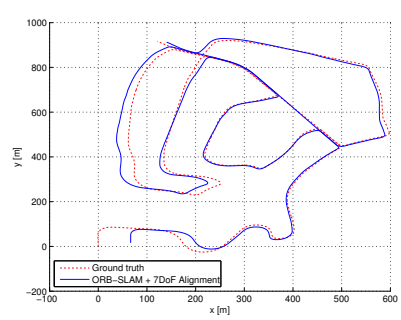

(a) Sequence 02

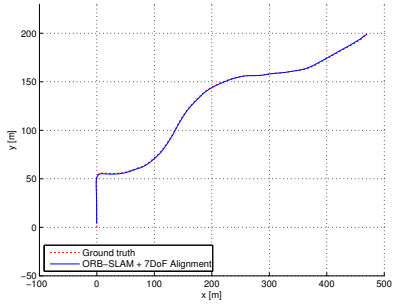

(b) Sequence 03

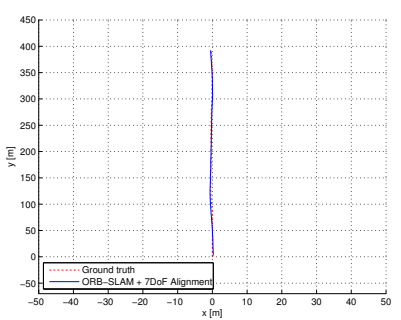

(c) Sequence 04

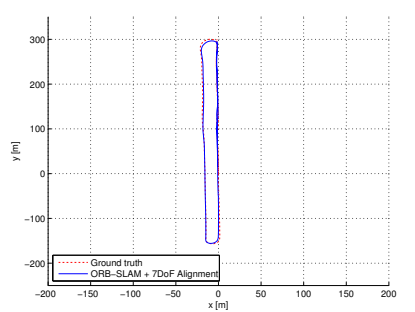

(d) Sequence 06

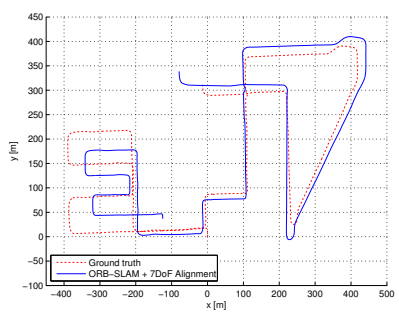

(e) Sequence 08

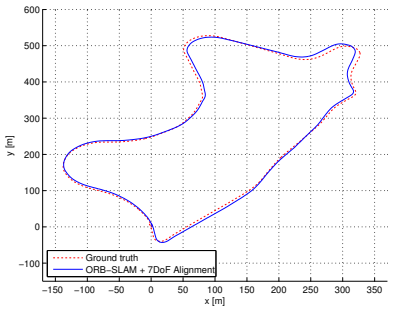

(f) Sequence 09

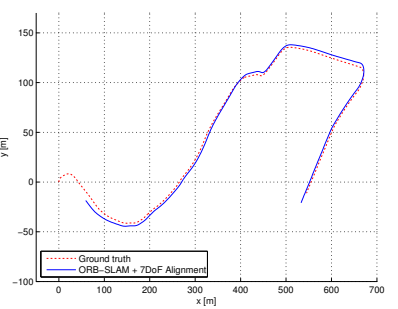

(g) Sequence 10

Fig. 12. ORB-SLAM keyframe trajectories in sequences $02,03,04,06,08,09$ and 10 from the odometry benchmark of the KITTI dataset. Sequence 08 does not contains loops and drift (especially scale) is not corrected. 
TABLE V

RESULTS OF OUR SYSTEM IN THE KITTI DATASET.

\begin{tabular}{|c|c|c|c|c|c|}
\cline { 3 - 6 } \multicolumn{2}{c|}{} & \multicolumn{2}{c|}{ ORB-SLAM } & \multicolumn{2}{c|}{ + Global BA (20 its. $)$} \\
\hline Sequence & $\begin{array}{c}\text { Dimension } \\
(\mathrm{m} \times \mathrm{m})\end{array}$ & KFs & $\begin{array}{c}\text { RMSE } \\
(\mathrm{m})\end{array}$ & $\begin{array}{c}\text { RMSE } \\
(\mathrm{m})\end{array}$ & $\begin{array}{c}\text { Time BA } \\
(\mathrm{s})\end{array}$ \\
\hline \hline KITTI 00 & $564 \times 496$ & 1391 & 6.68 & 5.33 & 24.83 \\
\hline KITTI 01 & $1157 \times 1827$ & $\mathrm{X}$ & $\mathrm{X}$ & $\mathrm{X}$ & $\mathrm{X}$ \\
\hline KITTI 02 & $599 \times 946$ & 1801 & 21.75 & 21.28 & 30.07 \\
\hline KITTI 03 & $471 \times 199$ & 250 & 1.59 & 1.51 & 4.88 \\
\hline KITTI 04 & $0.5 \times 394$ & 108 & 1.79 & 1.62 & 1.58 \\
\hline KITTI 05 & $479 \times 426$ & 820 & 8.23 & 4.85 & 15.20 \\
\hline KITTI 06 & $23 \times 457$ & 373 & 14.68 & 12.34 & 7.78 \\
\hline KITTI 07 & $191 \times 209$ & 351 & 3.36 & 2.26 & 6.28 \\
\hline KITTI 08 & $808 \times 391$ & 1473 & 46.58 & 46.68 & 25.60 \\
\hline KITTI 09 & $465 \times 568$ & 653 & 7.62 & 6.62 & 11.33 \\
\hline KITTI 10 & $671 \times 177$ & 411 & 8.68 & 8.80 & 7.64 \\
\hline
\end{tabular}

the trajectory error typically around the $1 \%$ of its dimensions, sometimes less as in sequence 03 with an error of the $0.3 \%$ or higher as in sequence 08 with the $5 \%$. In sequence 08 there are no loops and drift cannot be corrected, which makes clear the need of loop closures to achieve accurate reconstructions.

In this experiment we have also checked how much the reconstruction can be improved by performing 20 iterations of full $B A$, see the Appendix for details, at the end of each sequence. We have noticed that some iterations of full $B A$ slightly improves the accuracy in the trajectories with loops but it has negligible effect in open trajectories, which means that the output of our system is already very accurate. In any case if the most accurate results are needed our algorithm provides a set of matches, which define a strong camera network, and an initial guess, so that full $B A$ converge in few iterations.

Finally we wanted to show the efficacy of our loop closing approach and the influence of the $\theta_{\min }$ used to include edges in the essential graph. We have selected the sequence 09 (a very long sequence with a loop closure at the end), and in the same execution we have evaluated different loop closing strategies. In table VI we show the keyframe trajectory RMSE and the time spent in the optimization in different cases: without loop closing, if we directly apply a full $B A$ (20 or 100 iterations), if we apply only pose graph optimization (10 iterations with different number of edges) and if we apply pose graph optimization and full $B A$ afterwards. The results clearly show that before loop closure, the solution is so far from the optimal, that BA has convergence problems. Even after 100 iterations still the error is very high. On the other hand essential graph optimization shows fast convergence and more accurate results. It can be seen that the choice of $\theta_{\min }$ has not significant effect in accuracy but decreasing the number of edges the time can be significantly reduced. Performing an additional BA after the pose graph optimization slightly improves the accuracy while increasing substantially the time.
TABLE VI

COMPARISON OF LOOP CLOSING STRATEGIES IN KITTI 09

\begin{tabular}{|c|c|c|c|}
\hline Method & Time (s) & Pose Graph Edges & RMSE (m) \\
\hline \hline- & - & - & 48.77 \\
\hline BA (20) & 14.64 & - & 49.90 \\
\hline BA (100) & 72.16 & - & 18.82 \\
\hline EG (200) & 0.38 & 890 & 8.84 \\
\hline EG (100) & 0.48 & 1979 & 8.36 \\
\hline EG (50) & 0.59 & 3583 & 8.95 \\
\hline EG (15) & 0.94 & 6663 & 8.88 \\
\hline EG (100) + BA (20) & 13.40 & 1979 & 7.22 \\
\hline
\end{tabular}

First row shows results without loop closing. Number between brackets for BA (Bundle Adjustment) means number of Levenberg-Marquardt (LM) iterations, while for EG (Essential Graph) is the $\theta_{\min }$ to build the Essential Graph. All EG optimizations perform 10 LM iterations. (a) Without Loop Closing

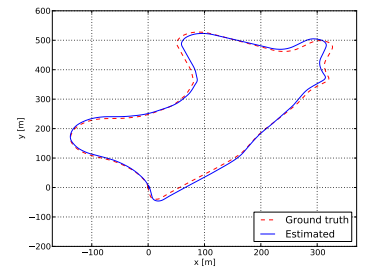

(c) EG (100)

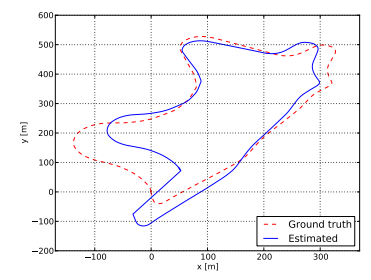

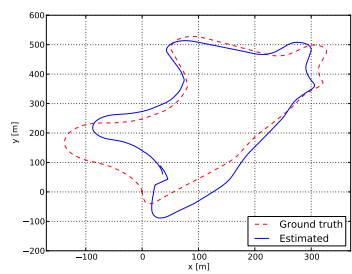

(b) BA (20)

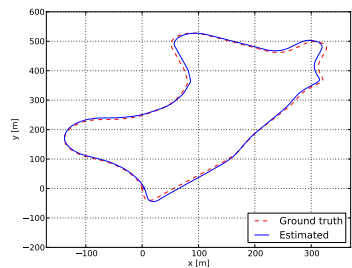

(d) $\mathrm{EG}(100)+\mathrm{BA}(20)$
Fig. 13. Comparison of different loop closing strategies in KITTI 09.

\section{CONCLUSIONS AND Discussion}

\section{A. Conclusions}

In this work we have presented a new monocular SLAM system with a detailed description of its building blocks and an exhaustive evaluation in public datasets. Our system has demonstrated that it can process sequences from indoor and outdoor scenes and from car, robot and hand-held motions. The accuracy of the system is typically below $1 \mathrm{~cm}$ in small indoor scenarios and of a few meters in large outdoor scenarios (once we have aligned the scale with the ground truth).

Currently PTAM by Klein and Murray [4] is considered the most accurate SLAM method from monocular video in real time. It is not coincidence that the backend of PTAM is bundle adjustment, which is well known to be the gold standard method for the offline Structure From Motion problem [2]. One of the main successes of PTAM, and the earlier work of Mouragnon [3], was to bring that knowledge into the robotics SLAM community and demonstrate its real time performance. 
The main contribution of our work is to expand the versatility of PTAM to environments that are intractable for that system. To achieve this, we have designed from scratch a new monocular SLAM system with some new ideas and algorithms, but also incorporating excellent works developed in the past few years, such as the loop detection of Gálvez-López and Tardós [5], the loop closing procedure and covisibility graph of Strasdat et.al [6], [7], the optimization framework g2o by Kuemmerle et. al [37] and ORB features by Rubble et. al [9]. To the best of our knowledge, no other system has demonstrated to work in as many different scenarios and with such accuracy. Therefore our system is currently the most reliable and complete solution for monocular SLAM. Our novel policy to spawn and cull keyframes, permits to create keyframes every few frames, which are eventually removed when considered redundant. This flexible map expansion is really useful in poorly conditioned exploration trajectories, i.e. close to pure rotations or fast movements. When operating repeatedly in the same environment, the map only grows if the visual content of the scene changes, storing a history of its different visual appearances. Interesting results for longterm mapping could be extracted analyzing this history.

Finally we have also demonstrated that ORB features have enough recognition power to enable place recognition from severe viewpoint change. Moreover they are so fast to extract and match (without the need of multi-threading or GPU acceleration) that enable real time accurate tracking and mapping.

\section{B. Sparse/Feature-based vs. Dense/Direct Methods}

Recent real-time monocular SLAM algorithms such as DTAM [44] and LSD-SLAM [10] are able to perform dense or semi dense reconstructions of the environment, while the camera is localized by optimizing directly over image pixel intensities. These direct approaches do not need feature extraction and thus avoid the corresponding artifacts. They are also more robust to blur, low-texture environments and highfrequency texture like asphalt [45]. Their denser reconstructions, as compared to the sparse point map of our system or PTAM, could be more useful for other tasks than just camera localization.

However, direct methods have their own limitations. Firstly, these methods assume a surface reflectance model that in real scenes produces its own artifacts. The photometric consistency limits the baseline of the matches, typically narrower than those that features allow. This has a great impact in reconstruction accuracy, which requires wide baseline observations to reduce depth uncertainty. Direct methods, if not correctly modeled, are quite affected by rolling-shutter, auto-gain and auto-exposure artifacts (as in the TUM RGB-D Benchmark). Finally, because direct methods are in general very computationally demanding, the map is just incrementally expanded as in DTAM, or map optimization is reduced to a pose graph, discarding all sensor measurements as in LSD-SLAM.

In contrast, feature-based methods are able to match features with a wide baseline, thanks to their good invariance to viewpoint and illumination changes. Bundle adjustment jointly optimizes camera poses and points over sensor measurements.
In the context of structure and motion estimation, Torr and Zisserman [46] already pointed the benefits of feature-based against direct methods. In this work we provide experimental evidence (see Section VIII-B) of the superior accuracy of feature-based methods in real-time SLAM. We consider that the future of monocular SLAM should incorporate the best of both approaches.

\section{Future Work}

The accuracy of our system can still be improved incorporating points at infinity in the tracking. These points, which are not seen with sufficient parallax and our system does not include in the map, are very informative of the rotation of the camera [21].

Another open way is to upgrade the sparse map of our system to a denser and more useful reconstruction. Thanks to our keyframe selection, keyframes comprise a compact summary of the environment with a very high pose accuracy and rich information of covisibility. Therefore the ORB-SLAM sparse map can be an excellent initial guess and skeleton, on top of which a dense and accurate map of the scene can be built. A first effort in this line is presented in [47].

\section{APPENDIX}

\section{NON-Linear Optimizations}

- Bundle Adjustment (BA) [1]: Map point 3D locations $\mathbf{X}_{w, j} \in \mathbb{R}^{3}$ and keyframe poses $\mathbf{T}_{i w} \in \mathrm{SE}(3)$, where $w$ stands for the world reference, are optimized minimizing the reprojection error with respect to the matched keypoints $\mathbf{x}_{i, j} \in \mathbb{R}^{2}$. The error term for the observation of a map point $j$ in a keyframe $i$ is:

$$
\mathbf{e}_{i, j}=\mathbf{x}_{i, j}-\pi_{i}\left(\mathbf{T}_{i w}, \mathbf{X}_{w, j}\right)
$$

where $\pi_{i}$ is the projection function:

$$
\begin{gathered}
\pi_{i}\left(\mathbf{T}_{i w}, \mathbf{X}_{w, j}\right)=\left[\begin{array}{c}
f_{i, u} \frac{x_{i, j}}{z_{i, j}}+c_{i, u} \\
f_{i, v} \frac{y_{i, j}}{z_{i, j}}+c_{i, v}
\end{array}\right] \\
{\left[\begin{array}{lll}
x_{i, j} & y_{i, j} & z_{i, j}
\end{array}\right]^{T}=\mathbf{R}_{i w} \mathbf{X}_{w, j}+\mathbf{t}_{i w}}
\end{gathered}
$$

where $\mathbf{R}_{i w} \in \mathrm{SO}(3)$ and $\mathbf{t}_{i w} \in \mathbb{R}^{3}$ are respectively the rotation and translation parts of $\mathbf{T}_{i w}$, and $\left(f_{i, u}, f_{i, v}\right)$ and $\left(c_{i, u}, c_{i, v}\right)$ are the focal length and principle point associated to camera $i$. The cost function to be minimized is:

$$
C=\sum_{i, j} \rho_{h}\left(\mathbf{e}_{i, j}^{T} \boldsymbol{\Omega}_{i, j}^{-1} \mathbf{e}_{i, j}\right)
$$

where $\rho_{h}$ is the Huber robust cost function and $\boldsymbol{\Omega}_{i, j}=$ $\sigma_{i, j}^{2} \mathbf{I}_{2 \times 2}$ is the covariance matrix associated to the scale at which the keypoint was detected. In case of full $B A$ (used in the map initialization explained in Section IV and in the experiments in Section VIII-E) we optimize all points and keyframes, by the exception of the first keyframe which remain fixed as the origin. In local $B A$ (see section VI-D) all points included in the local area are optimized, while a subset of keyframes is fixed. In pose optimization, or motion-only BA, (see section V) all points are fixed and only the camera pose is optimized. 
- Pose Graph Optimization over Sim(3) Constraints [6]: Given a pose graph of binary edges (see Section VII-D) we define the error in an edge as:

$$
\mathbf{e}_{i, j}=\log _{\operatorname{Sim}(3)}\left(\mathbf{S}_{i j} \mathbf{S}_{j w} \mathbf{S}_{i w}^{-1}\right)
$$

where $\mathbf{S}_{i j}$ is the relative $\operatorname{Sim}(3)$ transformation between both keyframes computed from the $\mathrm{SE}(3)$ poses just before the pose graph optimization and setting the scale factor to 1 . In the case of the loop closure edge this relative transformation is computed with the method of Horn [42]. The $\log _{\text {Sim3 } 3}$ [48] transforms to the tangent space, so that the error is a vector in $\mathbb{R}^{7}$. The goal is to optimize the $\operatorname{Sim}(3)$ keyframe poses minimizing the cost function:

$$
C=\sum_{i, j}\left(\mathbf{e}_{i, j}^{T} \mathbf{\Lambda}_{i, j} \mathbf{e}_{i, j}\right)
$$

where $\boldsymbol{\Lambda}_{i, j}$ is the information matrix of the edge, which, as in [48], we set to the identity. We fix the loop closure keyframe to fix the 7 degrees of gauge freedom. Although this method is a rough approximation of a full $B A$, we demonstrate experimentally in Section VIII-E that it has significantly faster and better convergence than BA.

- Relative Sim(3) Optimization:

Given a set of $n$ matches $i \Rightarrow j$ (keypoints and their associated 3D map points) between keyframe 1 and keyframe 2, we want to optimize the relative $\operatorname{Sim}(3)$ transformation $\mathbf{S}_{12}$ (see Section VII-B) that minimizes the reprojection error in both images:

$$
\begin{aligned}
& \mathbf{e}_{\mathbf{1}}=\mathbf{x}_{1, i}-\pi_{1}\left(\mathbf{S}_{12}, \mathbf{X}_{2, j}\right) \\
& \mathbf{e}_{\mathbf{2}}=\mathbf{x}_{2, j}-\pi_{2}\left(\mathbf{S}_{12}^{-1}, \mathbf{X}_{1, i}\right)
\end{aligned}
$$

and the cost function to minimize is:

$$
C=\sum_{n}\left(\rho_{h}\left(\mathbf{e}_{1}^{T} \boldsymbol{\Omega}_{1, i}^{-1} \mathbf{e}_{1}\right)+\rho_{h}\left(\mathbf{e}_{2}^{T} \boldsymbol{\Omega}_{2, j}^{-1} \mathbf{e}_{2}\right)\right)
$$

where $\boldsymbol{\Omega}_{1, i}$ and $\boldsymbol{\Omega}_{2, i}$ are the covariance matrices associated to the scale in which keypoints in image 1 and image 2 were detected. In this optimization the points are fixed.

\section{REFERENCES}

[1] B. Triggs, P. F. McLauchlan, R. I. Hartley, and A. W. Fitzgibbon, "Bundle adjustment a modern synthesis," in Vision algorithms: theory and practice, 2000, pp. 298-372.

[2] R. Hartley and A. Zisserman, Multiple View Geometry in Computer Vision, 2nd ed. Cambridge University Press, 2004.

[3] E. Mouragnon, M. Lhuillier, M. Dhome, F. Dekeyser, and P. Sayd, "Real time localization and 3d reconstruction," in Computer Vision and Pattern Recognition, 2006 IEEE Computer Society Conference on, vol. 1, 2006, pp. 363-370.

[4] G. Klein and D. Murray, "Parallel tracking and mapping for small AR workspaces," in IEEE and ACM International Symposium on Mixed and Augmented Reality (ISMAR), Nara, Japan, November 2007, pp. 225-234.

[5] D. Gálvez-López and J. D. Tardós, "Bags of binary words for fast place recognition in image sequences," IEEE Transactions on Robotics, vol. 28 , no. 5, pp. 1188-1197, 2012.

[6] H. Strasdat, J. M. M. Montiel, and A. J. Davison, "Scale drift-aware large scale monocular SLAM." in Robotics: Science and Systems (RSS), Zaragoza, Spain, June 2010.

[7] H. Strasdat, A. J. Davison, J. M. M. Montiel, and K. Konolige, "Double window optimisation for constant time visual SLAM," in IEEE International Conference on Computer Vision (ICCV), Barcelona, Spain, November 2011, pp. 2352-2359.
[8] C. Mei, G. Sibley, and P. Newman, "Closing loops without places," in IEEE/RSJ International Conference on Intelligent Robots and Systems (IROS), Taipei, Taiwan, October 2010, pp. 3738-3744.

[9] E. Rublee, V. Rabaud, K. Konolige, and G. Bradski, "ORB: an efficient alternative to SIFT or SURF," in IEEE International Conference on Computer Vision (ICCV), Barcelona, Spain, November 2011, pp. 25642571.

[10] J. Engel, T. Schöps, and D. Cremers, "LSD-SLAM: Large-scale direct monocular SLAM," in European Conference on Computer Vision (ECCV), Zurich, Switzerland, September 2014, pp. 834-849.

[11] R. Mur-Artal and J. D. Tardós, "Fast relocalisation and loop closing in keyframe-based SLAM," in IEEE International Conference on Robotics and Automation (ICRA), Hong Kong, China, June 2014, pp. 846-853.

[12] — "ORB-SLAM: Tracking and mapping recognizable features," in MVIGRO Workshop at Robotics Science and Systems (RSS), Berkeley, USA, July 2014.

[13] B. Williams, M. Cummins, J. Neira, P. Newman, I. Reid, and J. D. Tardós, "A comparison of loop closing techniques in monocular SLAM," Robotics and Autonomous Systems, vol. 57, no. 12, pp. 1188-1197, 2009.

[14] D. Nister and H. Stewenius, "Scalable recognition with a vocabulary tree," in IEEE Computer Society Conference on Computer Vision and Pattern Recognition (CVPR), vol. 2, New York City, USA, June 2006, pp. 2161-2168.

[15] M. Cummins and P. Newman, "Appearance-only SLAM at large scale with FAB-MAP 2.0," The International Journal of Robotics Research, vol. 30, no. 9, pp. 1100-1123, 2011.

[16] M. Calonder, V. Lepetit, C. Strecha, and P. Fua, "BRIEF: Binary Robust Independent Elementary Features," in European Conference on Computer Vision (ECCV), Hersonissos, Greece, September 2010, pp. $778-792$.

[17] E. Rosten and T. Drummond, "Machine learning for high-speed corner detection," in European Conference on Computer Vision (ECCV), Graz, Austria, May 2006, pp. 430-443.

[18] H. Bay, T. Tuytelaars, and L. Van Gool, "SURF: Speeded Up Robust Features," in European Conference on Computer Vision (ECCV), Graz, Austria, May 2006, pp. 404-417.

[19] D. G. Lowe, "Distinctive image features from scale-invariant keypoints," International Journal of Computer Vision, vol. 60, no. 2, pp. 91-110, 2004.

[20] A. J. Davison, I. D. Reid, N. D. Molton, and O. Stasse, "MonoSLAM: Real-time single camera SLAM," IEEE Transactions on Pattern Analysis and Machine Intelligence, vol. 29, no. 6, pp. 1052-1067, 2007.

[21] J. Civera, A. J. Davison, and J. M. M. Montiel, "Inverse depth parametrization for monocular SLAM," IEEE Transactions on Robotics, vol. 24, no. 5, pp. 932-945, 2008.

[22] C. Forster, M. Pizzoli, and D. Scaramuzza, "SVO: Fast semi-direct monocular visual odometry," in Proc. IEEE Intl. Conf. on Robotics and Automation, Hong Kong, China, June 2014, pp. 15-22.

[23] O. D. Faugeras and F. Lustman, "Motion and structure from motion in a piecewise planar environment," International Journal of Pattern Recognition and Artificial Intelligence, vol. 2, no. 03, pp. 485-508, 1988.

[24] W. Tan, H. Liu, Z. Dong, G. Zhang, and H. Bao, "Robust monocular SLAM in dynamic environments," in IEEE International Symposium on Mixed and Augmented Reality (ISMAR), Adelaide, Australia, October 2013, pp. 209-218.

[25] H. Lim, J. Lim, and H. J. Kim, "Real-time 6-DOF monocular visual SLAM in a large-scale environment," in IEEE International Conference on Robotics and Automation (ICRA), Hong Kong, China, June 2014, pp. $1532-1539$.

[26] D. Nistér, "An efficient solution to the five-point relative pose problem," IEEE Transactions on Pattern Analysis and Machine Intelligence, vol. 26, no. 6, pp. 756-770, 2004.

[27] H. Longuet-Higgins, "The reconstruction of a plane surface from two perspective projections," Proceedings of the Royal Society of London. Series B. Biological Sciences, vol. 227, no. 1249, pp. 399-410, 1986.

[28] P. H. Torr, A. W. Fitzgibbon, and A. Zisserman, "The problem of degeneracy in structure and motion recovery from uncalibrated image sequences," International Journal of Computer Vision, vol. 32, no. 1, pp. 27-44, 1999.

[29] A. Chiuso, P. Favaro, H. Jin, and S. Soatto, "Structure from motion causally integrated over time," IEEE Transactions on Pattern Analysis and Machine Intelligence, vol. 24, no. 4, pp. 523-535, 2002.

[30] E. Eade and T. Drummond, "Scalable monocular SLAM," in IEEE Computer Society Conference on Computer Vision and Pattern Recognition (CVPR), vol. 1, New York City, USA, June 2006, pp. 469-476.

[31] H. Strasdat, J. M. M. Montiel, and A. J. Davison, "Visual SLAM: Why filter?" Image and Vision Computing, vol. 30, no. 2, pp. 65-77, 2012. 
[32] G. Klein and D. Murray, "Improving the agility of keyframe-based slam," in European Conference on Computer Vision (ECCV), Marseille, France, October 2008, pp. 802-815.

[33] K. Pirker, M. Ruther, and H. Bischof, "CD SLAM-continuous localization and mapping in a dynamic world," in IEEE/RSJ International Conference on Intelligent Robots and Systems (IROS), San Francisco, USA, September 2011, pp. 3990-3997.

[34] S. Song, M. Chandraker, and C. C. Guest, "Parallel, real-time monocular visual odometry," in IEEE International Conference on Robotics and Automation (ICRA), 2013, pp. 4698-4705.

[35] P. F. Alcantarilla, J. Nuevo, and A. Bartoli, "Fast explicit diffusion for accelerated features in nonlinear scale spaces," in British Machine Vision Conference (BMVC), Bristol, UK, 2013.

[36] X. Yang and K.-T. Cheng, "LDB: An ultra-fast feature for scalable augmented reality on mobile devices," in IEEE International Symposium on Mixed and Augmented Reality (ISMAR), 2012, pp. 49-57.

[37] R. Kuemmerle, G. Grisetti, H. Strasdat, K. Konolige, and W. Burgard, "g2o: A general framework for graph optimization," in IEEE International Conference on Robotics and Automation (ICRA), Shanghai, China, May 2011, pp. 3607-3613.

[38] J. Sturm, N. Engelhard, F. Endres, W. Burgard, and D. Cremers, "A benchmark for the evaluation of RGB-D SLAM systems," in IEEE/RSJ International Conference on Intelligent Robots and Systems (IROS), Vilamoura, Portugal, October 2012, pp. 573-580.

[39] M. Smith, I. Baldwin, W. Churchill, R. Paul, and P. Newman, "The new college vision and laser data set," The International Journal of Robotics Research, vol. 28, no. 5, pp. 595-599, 2009.

[40] A. Geiger, P. Lenz, C. Stiller, and R. Urtasun, "Vision meets robotics: The KITTI dataset," The International Journal of Robotics Research, vol. 32, no. 11, pp. 1231-1237, 2013.

[41] V. Lepetit, F. Moreno-Noguer, and P. Fua, "EPnP: An accurate O(n) solution to the PnP problem," International Journal of Computer Vision, vol. 81, no. 2, pp. 155-166, 2009.

[42] B. K. P. Horn, "Closed-form solution of absolute orientation using unit quaternions," Journal of the Optical Society of America A, vol. 4, no. 4, pp. 629-642, 1987.

[43] F. Endres, J. Hess, J. Sturm, D. Cremers, and W. Burgard, "3-d mapping with an rgb-d camera," IEEE Transactions on Robotics, vol. 30, no. 1 , pp. 177-187, 2014

[44] R. A. Newcombe, S. J. Lovegrove, and A. J. Davison, "DTAM: Dense tracking and mapping in real-time," in IEEE International Conference on Computer Vision (ICCV), Barcelona, Spain, November 2011, pp. 2320 2327.

[45] S. Lovegrove, A. J. Davison, and J. Ibanez-Guzmán, "Accurate visual odometry from a rear parking camera," in IEEE Intelligent Vehicles Symposium (IV), 2011, pp. 788-793.

[46] P. H. Torr and A. Zisserman, "Feature based methods for structure and motion estimation," in Vision Algorithms: Theory and Practice. Springer, 2000, pp. 278-294.

[47] R. Mur-Artal and J. D. Tardos, "Probabilistic semi-dense mapping from highly accurate feature-based monocular SLAM," in Robotics: Science and Systems (RSS), Rome, Italy, July 2015.

[48] H. Strasdat, "Local Accuracy and Global Consistency for Efficient Visual SLAM," Ph.D. dissertation, Imperial College, London, October 2012.

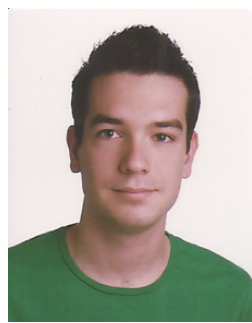

Raúl Mur Artal was born in Zaragoza, Spain in 1989. He received the Industrial Engineering degree (mention in Industrial Automation and Robotics) in 2012 and the M.S. degree in Systems and Computer Engineering in 2013 from the University of Zaragoza, where he is currently working towards the $\mathrm{PhD}$. degree with the I3A Robotics, Perception and Real-Time Group.

His research interests include Visual Localization and Long-Term Mapping.

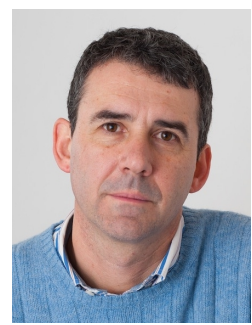

J. M . M. Montiel was born in Arnedo, Spain, in 1967. He received the M.S. and Ph.D. degrees in electrical engineering from the Universidad de Zaragoza, Spain, in 1992 and 1996, respectively. $\mathrm{He}$ is currently a Full Professor with the Departamento de Informática e Ingeniería de Sistemas, Universidad de Zaragoza, where he is in charge of Perception and Computer Vision research grants and courses. His current interests include, real-time vision localization and semantic mapping for rigid and non rigid environments, and the transference of this technology to robotic and nonrobotic application domains. Prof. Martínez Montiel is a member of the I3A Robotics, Perception, and Real-Time Group. $\mathrm{He}$ has been awarded several Spanish MEC grants to fund research at the University of Oxford, UK, and at Imperial College London, UK.

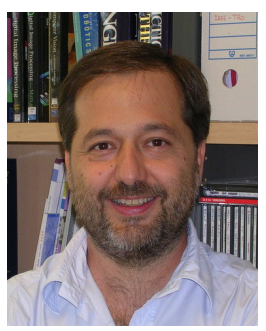

Juan D. Tardós was born in Huesca, Spain, in 1961. He earned the M.S. and Ph.D. degrees in electrical engineering from the University of Zaragoza, Spain, in 1985 and 1991, respectively. He is Full Professor with the Departamento de Informática e Ingeniería de Sistemas, University of Zaragoza, where he is in charge of courses in robotics, computer vision, and artificial intelligence. His research interests include SLAM, perception and mobile robotics. Prof. Tardós is a member of the I3A Robotics, Perception, and Real-Time Group 